\title{
BCR-ABL Tyrosine Kinase Inhibitors: Which Mechanism(s) May Explain the Risk of Thrombosis?
}

\author{
Hélène Haguet ${ }^{1,2}$ Jonathan Douxfils ${ }^{1,4}$ Christian Chatelain $^{1}$ Carlos Graux ${ }^{3}$ François Mullier ${ }^{2}$ \\ Jean-Michel Dogné ${ }^{1}$
}

1 University of Namur, Namur Thrombosis and Hemostasis Center (NTHC), Namur Research Institute for Life Sciences (NARILIS), Department of Pharmacy, Namur, Belgium

2 Université catholique de Louvain, CHU UCL Namur, Namur Thrombosis and Hemostasis Center, Hematology Laboratory, Yvoir, Belgium

${ }^{3}$ Université catholique de Louvain, CHU UCL Namur, Namur Thrombosis and Hemostasis Center, Department of Hematology, Yvoir, Belgium

${ }^{4}$ QUALIblood s.a., Namur, Belgium

TH Open 2018;2:e68-e88.

\begin{abstract}
Address for correspondence Hélène Haguet, MBS, Department of Pharmacy, Namur Thrombosis and Hemostasis Center (NTHC), Namur Research Institute for Life Sciences (NARILIS), University of Namur, Rue de Bruxelles, 61, B-5000 Namur, Belgium (e-mail: helene.haguet@gmail.com).
\end{abstract}

\begin{abstract}
Keywords

- BCR-ABL

- arterial thrombotic events

- tyrosine kinase inhibitors

- chronic myeloid leukemia

Imatinib, the first-in-class BCR-ABL tyrosine kinase inhibitor (TKI), had been a revolution for the treatment of chronic myeloid leukemia (CML) and had greatly enhanced patient survival. Second- (dasatinib, nilotinib, and bosutinib) and third-generation (ponatinib) TKIs have been developed to be effective against BCR-ABL mutations making imatinib less effective. However, these treatments have been associated with arterial occlusive events. This review gathers clinical data and experiments about the pathophysiology of these arterial occlusive events with BCR-ABL TKIs. Imatinib is associated with very low rates of thrombosis, suggesting a potentially protecting cardiovascular effect of this treatment in patients with BCR-ABLCML. This protective effect might be mediated by decreased platelet secretion and activation, decreased leukocyte recruitment, and anti-inflammatory or antifibrotic effects. Clinical data have guided mechanistic studies toward alteration of platelet functions and atherosclerosis development, which might be secondary to metabolism impairment. Dasatinib, nilotinib, and ponatinib affect endothelial cells and might induce atherogenesis through increased vascular permeability. Nilotinib also impairs platelet functions and induces hyperglycemia and dyslipidemia that might contribute to atherosclerosis development. Description of the pathophysiology of arterial thrombotic events is necessary to implement risk minimization strategies.
\end{abstract}

\section{Introduction}

In 2001, the approval of imatinib, the first-in-class tyrosine kinase inhibitor (TKI) targeting BCR-ABL, transformed the prognosis of patients with chronic-phase $(\mathrm{CP})$ chronic myeloid leukemia (CML) from a life-threatening condition to a manageable and chronic disease. ${ }^{1}$ Yet, despite satisfactory outcomes, $33 \%$ of patients did not achieved optimal response because of treatment resistance or intolerance. ${ }^{1}$ The identi-

received

September 13, 2017

accepted after revision

November 27, 2017
DOI https://doi.org/

10.1055/s-0038-1624566. ISSN 2512-9465. fication of the predominant resistance mechanism (i.e., point mutations in the kinase domain of Bcr-Abl) led to the development of second-generation BCR-ABL TKIs (dasatinib, nilotinib, and bosutinib, respectively, approved in 2006, 2007, and 2012) active against most of the BCR-ABL mutated forms. ${ }^{2,3}$ Second-generation TKIs demonstrated no or little improvement of the overall survival compared with imatinib, ${ }^{4-6}$ but two of these (i.e., dasatinib and nilotinib) improve
License terms

Stuttgart · New York

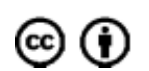


surrogate outcomes and permit quicker and deeper achievement of molecular response, which is criteria to try treatment cessation (i.e., $\mathrm{MR}^{4}$ or higher molecular response stable for at least 2 years). ${ }^{7}$ Based on these results, dasatinib and nilotinib were approved in 2010 for frontline management of CML, whereas bosutinib is used only after failure or intolerance of first-line BCR-ABL TKIs. Unfortunately, these treatments were ineffective against a common mutation (14\% of all mutations) in the gatekeeper residue of BCR-ABL (i.e., the T315I ${ }^{\mathrm{a}}$ mutation), ${ }^{8-10}$ requiring the development of a thirdgeneration TKI (ponatinib), efficient against this mutation. Ponatinib is currently the only treatment active against the T315I mutation and is therefore reserved for patients with this mutation or for patients resistant to frontline treatments. $^{11}$

Since its approval, the first-generation TKI, imatinib, has demonstrated reassuring safety profile, with low rate of grade $3 / 4$ adverse events and excellent tolerability. ${ }^{12,13}$ Conversely, new-generation BCR-ABL TKIs-nilotinib, dasatinib, bosutinib, and ponatinib-are more recent and display different safety profile. Dasatinib, nilotinib, and ponatinib are largely associated with fluid retention and dasatinib specifically induces high rate of pleural effusions. ${ }^{14-18}$ Nilotinib induces metabolic disorders such as dyslipidemia and hyperglycemia, whereas bosutinib safety profile is mainly characterized by gastrointestinal events (i.e., diarrhea, nausea, vomiting). ${ }^{19,20}$ Finally, ponatinib has been rapidly associated with high rate of vascular occlusion. ${ }^{21}$

Recently, meta-analyses of randomized clinical trials established that ponatinib is not the only new-generation TKI that increases the cardiovascular risk. ${ }^{22,23}$ The four newgeneration BCR-ABL TKIs increase the risk of vascular occlusive events compared with imatinib, especially arterial occlusive diseases, and this is in accordance with clinical trial data. ${ }^{22-25}$ However, this cardiovascular risk is controversy for dasatinib because of the low incidence ( 1.1 per 100 patient-year) of cardiovascular events in clinical trials. ${ }^{26,27}$ Recently, a large retrospective analysis of CP-CML patients treated with BCR-ABL TKIs at the MD Anderson Cancer Center confirmed the increased risk of vascular occlusive events with dasatinib. ${ }^{28}$ Another controversial point is the effect of imatinib on the cardiovascular system. Indeed, imatinib is associated with low risk of cardiovascular events and it was therefore hypothesized that imatinib prevents their occurrence. ${ }^{29,30}$ Clinical data indicate that most patients developing arterial occlusive events with new-generation BCR-ABL TKIs are high-risk patients, but cardiovascular events also occurred in young and healthy patients. Additional information on clinical safety of BCR-ABL TKIs is described in the Supplementary Material (-Table S1). We assume that the mechanism underlying arterial thrombosis with BCR-ABL TKIs might be multiple. The predominance of arterial events raised concerns about the impact of BCR-ABL TKIs on platelet

\footnotetext{
a T315I: Substitution at position 315 in BCR-ABL from a threonine to an isoleucine. This substitution alters the structure of the ATPbinding pocket and eliminates a crucial hydrogen bond required for binding of first- and second-generation TKIs.
}

functions, atherosclerosis, and metabolism, and precluded prothrombotic states to be responsible of these events. ${ }^{31}$

This review particularly focuses on the contribution of glucose and lipid metabolism, atherosclerosis, and platelets in the occurrence of cardiovascular events with new-generation TKIs. The last section discusses relevant off-targets that might be implicated in the cardiovascular toxicity. The discovery of the mechanism(s) by which arterial occlusive events arose in CML patients would help in the management of patients treated with BCR-ABL TKIs and implement risk minimization measures. Discovery of the pathophysiology of these events in CML patients might also led to the development of predictive biomarkers or to the development of new therapies with no or reduced cardiovascular toxicity profile while keeping an unaltered efficacy.

\section{Impact on Platelet Functions}

BCR-ABL TKIs are associated with both bleeding and thrombotic complications. - Table 1 describes experiments assessing the impact of BCR-ABL TKIs on platelet production and functions. Imatinib and dasatinib induce hemorrhagic events in patients with CML. Interestingly, dasatinib-associated hemorrhages occurred both in patients with and without thrombocytopenia. ${ }^{32}$ In vitro and in vivo investigations demonstrated that dasatinib affects both platelet functions (i.e., platelet aggregation, secretion, and activation) and platelet formation by impairment of megakaryocyte migration. ${ }^{33-36}$ Furthermore, dasatinib decreases thrombus formation in vitro, in vivo, and ex vivo, ${ }^{34}$ and decreases the number of procoagulant platelets (i.e., phosphatidylserineexposing platelets). ${ }^{35}$ Several dasatinib off-targets are implicated in platelet signaling and functions including members of the SFKs (e.g., Src, Lyn, Fyn, Lck, and Yes) (- Fig. 1). ${ }^{37,38}$ However, SFKs are also inhibited by bosutinib without disturbance of platelet aggregation and adhesion. Dasatinib also inhibits Syk, BTK, and members of the ephrin family ${ }^{\mathrm{b}}$ (e.g., EphA2), all known to be involved in platelet functions.

Experimental assessments of platelet functions with imatinib demonstrate less pronounced effects on platelets. Imatinib inhibits platelet aggregation only at high doses, ${ }^{34}$ and does not interfere with platelet aggregation in vivo. ${ }^{39}$ However, in vitro studies also indicate decreased platelet secretion and activation by imatinib. ${ }^{34}$ The mechanism by which imatinib inhibits platelet functions is unknown. Oppositely to dasatinib, imatinib does not inhibit SFKs, ephrins, BTK, and Syk. A hypothesis also suggests that imatinib induces bleeding disorders because of BCR-ABL rearrangements in megakaryocytic cell lines, leading to clonal expansion of dysfunctional megakaryocytes. ${ }^{40}$

Even if ponatinib induces very few bleeding disorders, assessment of primary hemostasis in CML patients demonstrated that ponatinib induces defect in platelet aggregation. This impairment was found at all ponatinib dosage, in patients with or without low platelet counts. ${ }^{41}$ These results

\footnotetext{
b Ephrin family: Members of this family are involved in platelet spreading, adhesion to fibrinogen and platelet secretion.
} 


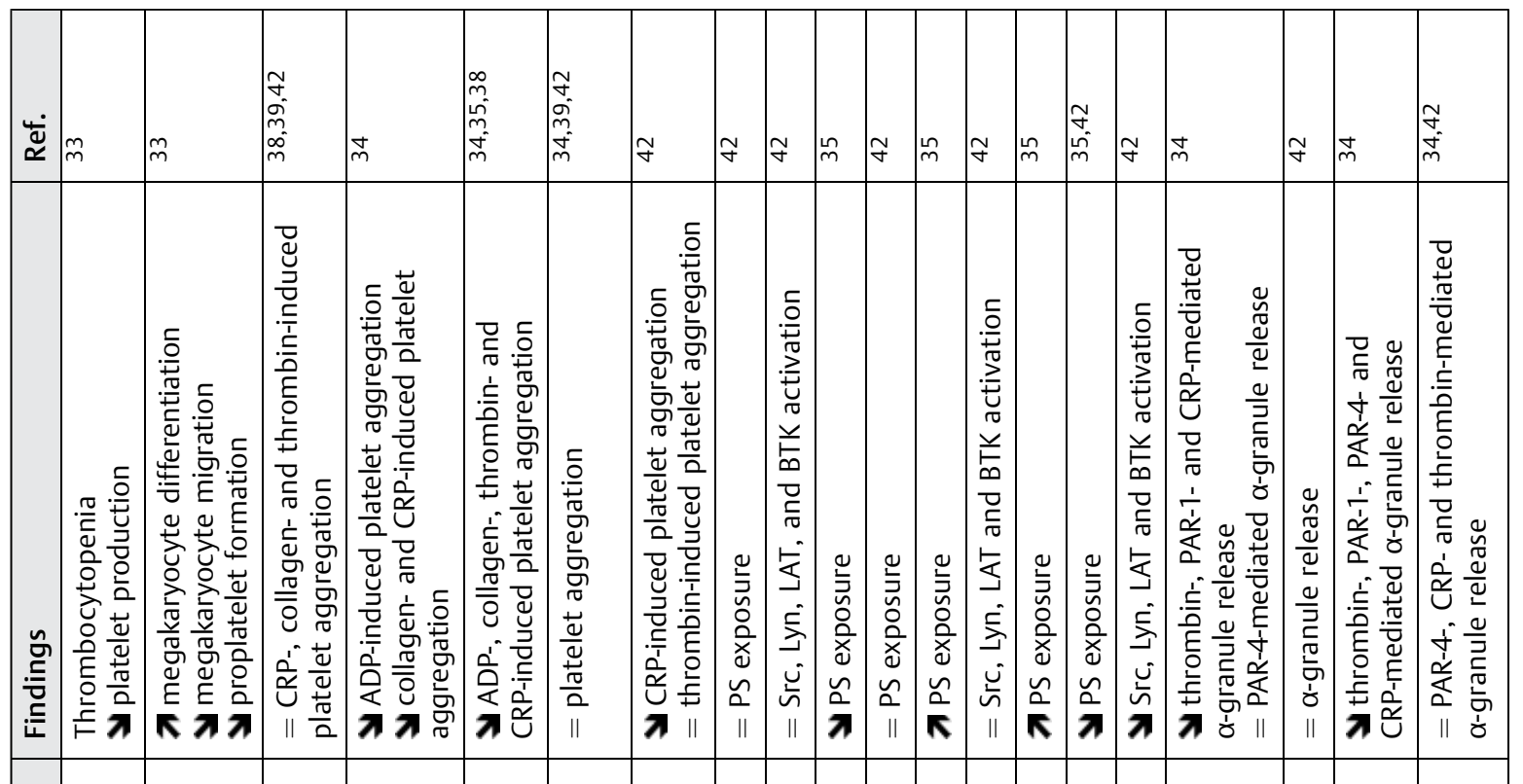

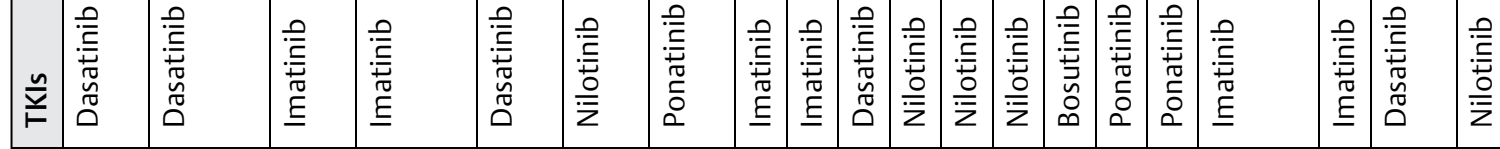

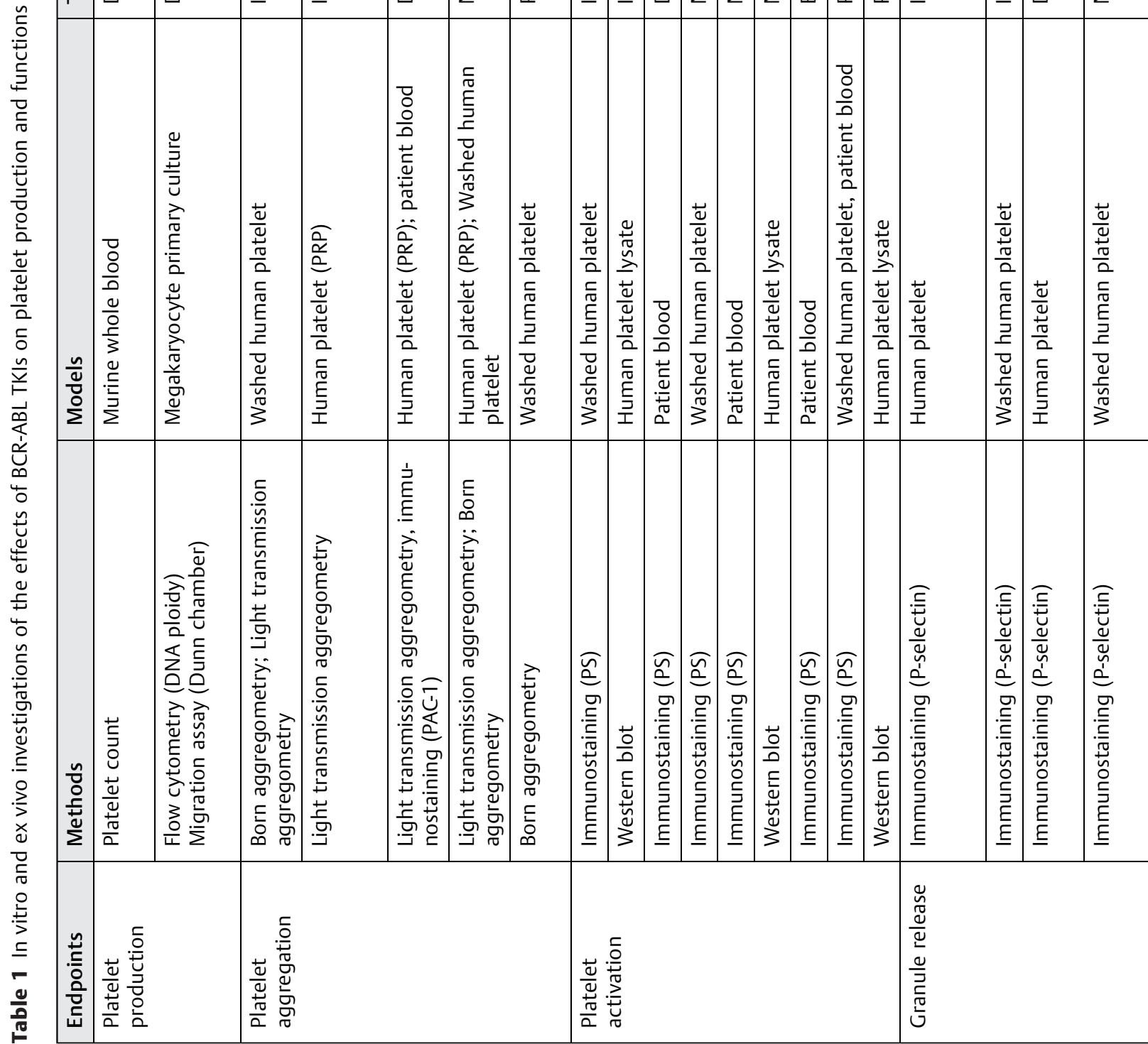




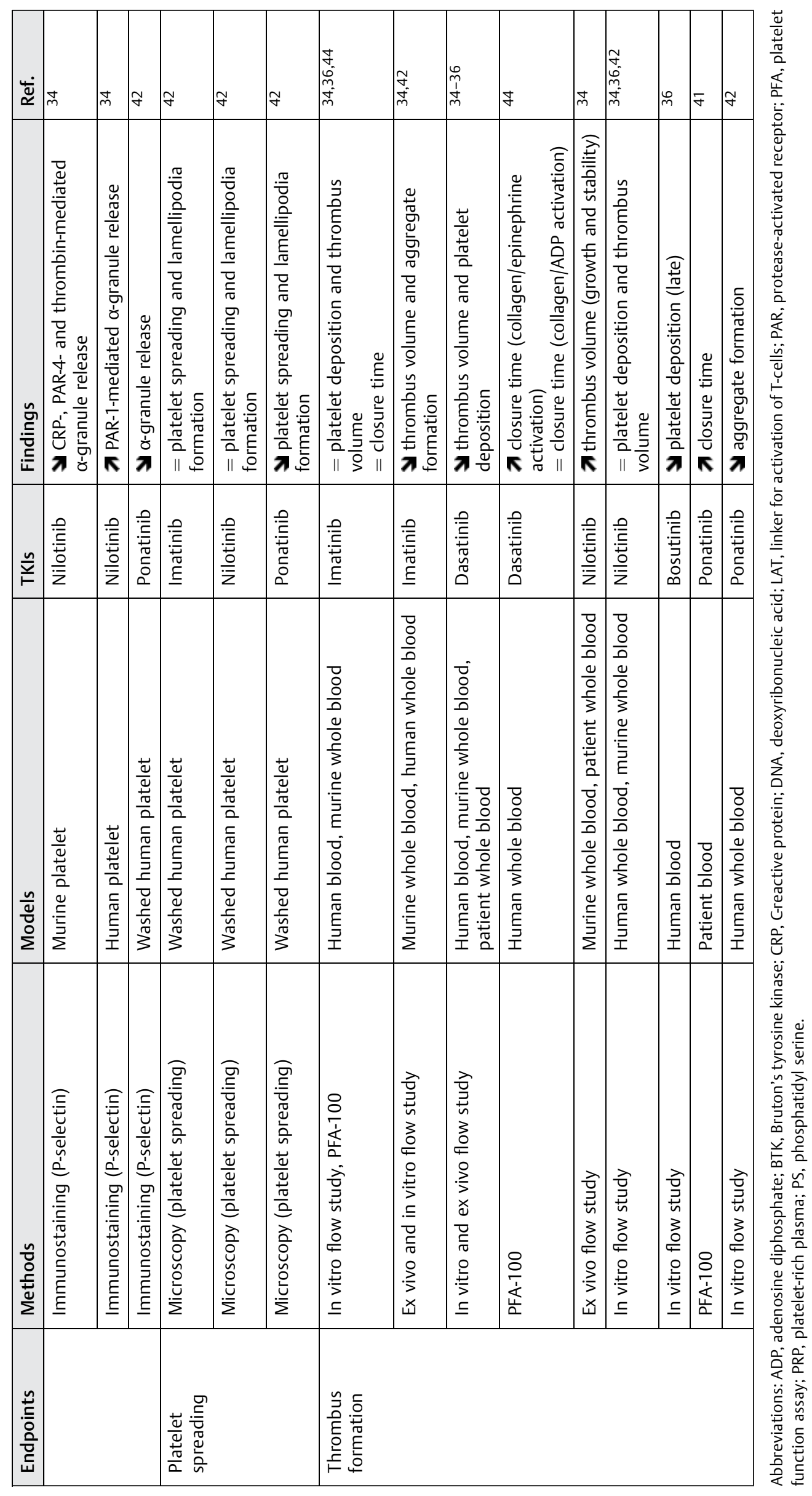




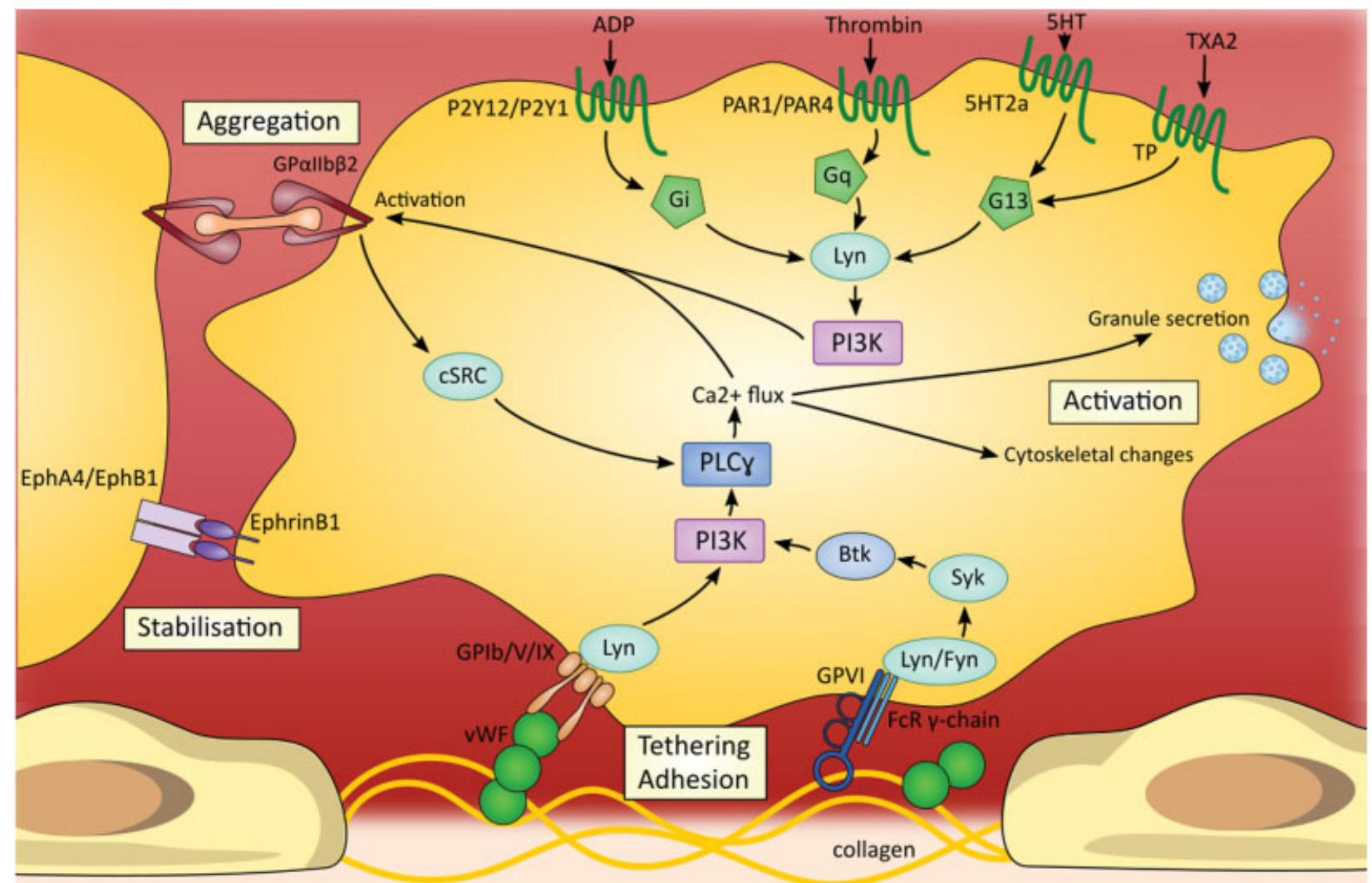

Fig. 1 Signaling pathways supporting platelet adhesion, activation, and aggregation. Tyrosine kinases are involved in several pathways and contribute to platelet adhesion, aggregation, and activation. Important players in platelet signaling are members of the Src family kinases; particularly Lyn, Fyn, and cSRC. These three tyrosine kinases are inhibited by dasatinib which might explain platelet dysfunction encountered with this treatment. Additionally, dasatinib also inhibits BTK, Syk, EphA4, and EphB1-four tyrosine kinases involved in platelet activation and aggregate stabilization. 5HT, 5-hydroxytryptamine; ADP, adenosine diphosphate; Btk, Bruton's tyrosine kinase; Ca, calcium; Eph, ephrin; FcR, Fc receptor; GP, glycoprotein; PAR, protease-activated receptor; PI3K, phosphoinositide 3-kinase; PLC, phospholipase C; TXA2, thromboxane A2; vWF, Von Willebrand factor.

were in accordance with in vitro studies which previously demonstrated similar characteristics than dasatinib (i.e., decrease of platelet spreading, aggregation, P-selectin secretion, and phosphatidylserine exposure). ${ }^{35,42}$ However, in vitro assays tested ponatinib at $1 \mu \mathrm{M}$, a dose far higher than the concentration observed in patients on treatment. ${ }^{43}$ Nilotinib and bosutinib are not associated with bleeding disorders in CML patients. First in vitro studies demonstrated little or no effect on platelet aggregation and activation with these two TKIs. ${ }^{36,39,44}$ However, recent experiments described prothrombotic phenotype of platelets induced by nilotinib, with increase of PAR- $1^{\mathrm{c}}$-mediated platelet secretion, adhesion, and activation, without disturbing platelet aggregation. ${ }^{34}$ Additional studies demonstrated that nilotinib increases secretion of adhesive molecules as well as thrombus formation and stability ex vivo. ${ }^{34}$

To summarize, dasatinib and imatinib induce hemorrhagic events through alteration of platelet functions, but the

\footnotetext{
C PAR-1: protease-activated receptor 1. PAR receptors mediate cellular effects of thrombin in platelets and endothelial cells.
}

molecular mechanism needs to be better determined. Ponatinib also impairs platelet functions. Therefore, no current data involve platelets in the pathogenesis of arterial thrombosis occurring with dasatinib and ponatinib. Oppositely, nilotinib might induce arterial thrombosis through alteration of platelet secretion, adhesion, and activation.

\section{Metabolic Dysregulation}

\section{Glucose Metabolism}

BCR-ABL TKIs have contradictory effect on glucose metabolism. Imatinib and dasatinib improve glucose metabolism and type 2 diabetes management in CML patients (i.e., decrease of antidiabetic drug dosage and reversal of type 2 diabetes). ${ }^{14,45-49}$ This clinical profile is in accordance with in vivo studies in which imatinib is effective to prevent the development of type 1 diabetes in prediabetic mice, without impacting the adaptive immune system. ${ }^{50}$ Therefore, imatinib is currently tested in clinical trials for patients suffering from type 1 diabetes mellitus (NCT01781975). The mechanism(s) by which dasatinib and imatinib improve glucose 


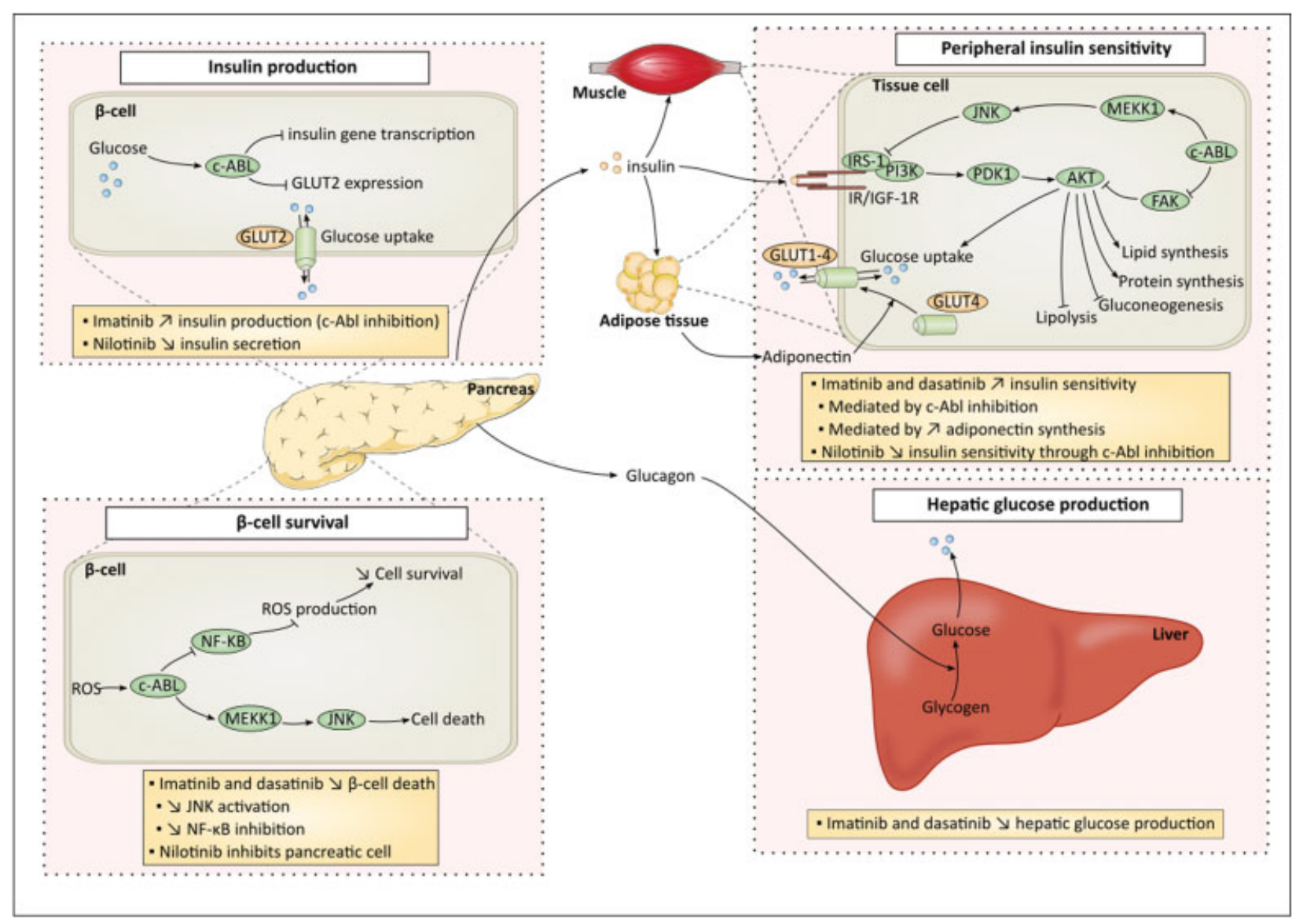

Fig. 2 Effects of BCR-ABL TKIs on glucose metabolism. Imatinib and dasatinib possess hypoglycemic effects, whereas nilotinib increases blood glucose level and diabetes development. The figure describes glucose metabolism and boxes contain emitted hypotheses for effects of imatinib, dasatinib, and nilotinib on glucose metabolism. Four major hypotheses have been emitted including impact on insulin production by $\beta$-cells, $\beta$ cell survival, peripheral insulin sensitivity, and hepatic glucose production. ABL, Abelson; FAK, focal adhesion kinase; GLUT, glucose transporter; IRS-1, insulin receptor substrate 1; JNK, c-Jun N-terminal kinases; MEKK1, MAPK/ERK kinase kinase 1; NF-KB, nuclear factor-kappa B; PDK1, pyruvate dehydrogenase kinase 1; PI3K, phosphoinositide 3-kinase; ROS, reactive oxygen species.

metabolism remains unknown. Global hypotheses suggest that imatinib increases peripheral insulin sensitivity, promotes $\beta$-cell survival, or decreases hepatic glucose production (-Fig. 2). ${ }^{51-54}$ This latter hypothesis (i.e., decreased hepatic glucose production by imatinib) is not currently the preferred theory, whereas it was demonstrated that imatinib weakly affects hepatic glucose production. ${ }^{51} \mathrm{Sev}-$ eral targets might be involved in this metabolic effect. PDGFR has already been linked with type 1 diabetes reversal. ${ }^{50}$ Hägerkvist et al hypothesized that c-Abl inhibition by imatinib promotes $\beta$-cell survival through activation of NF-KB signaling and inhibition of proapoptotic pathways (-Fig. 2). ${ }^{53,54}$ Inhibition of $\mathrm{c}-\mathrm{Abl}$ in $\beta$-cells might also increase insulin production and contribute to the glucose regulation by imatinib. ${ }^{55}$ It was also speculated that imatinib decreases insulin resistance in peripheral tissues due to cAbl-dependent JNK inactivation. ${ }^{d, 51}$ Similar hypotheses might be translated to dasatinib because of the similar offtarget inhibitory profile (i.e., dasatinib also inhibits c-Abl and

d JNK: c-Jun N-terminal kinases. JNK is responsive to stress stimuli and mediates insulin resistance through inhibition of insulin receptor substrate.
PDGFR). It was hypothesized that imatinib and dasatinib impact glucose metabolism through reduced adipose mass. ${ }^{51,56}$ However, clinical data do not demonstrate weight loss in CML patients and do not favor this hypothesis. In both imatinib- and dasatinib-treated patients, increased circulating adiponectin ${ }^{\mathrm{e}}$ level correlates with decreased insulin resistance. ${ }^{57,58}$ This correlation might be explained by the translocation of the glucose transporter GLUT4 $4^{\mathrm{f}}$ from the cytoplasm to the cell membrane following adiponectin signaling. ${ }^{59}$ Additionally, adiponectin has been related to decreased hepatic glucose production which could be an additional mechanism by which imatinib and dasatinib improve glucose metabolism. ${ }^{60}$ It was speculated that the raise of adiponectin level with imatinib and dasatinib is the consequence of increased adipogenesis subsequent to PDGFR inhibition. ${ }^{61}$

\footnotetext{
e Adiponectin is a protein regulating glucose metabolism. Adiponectin increases peripheral insulin sensitivity by improving glucose uptake.

f GLUT4: Glucose transporter type 4. GLUT4 is an insulin-regulated glucose transporter expressed in peripheral tissues.
} 


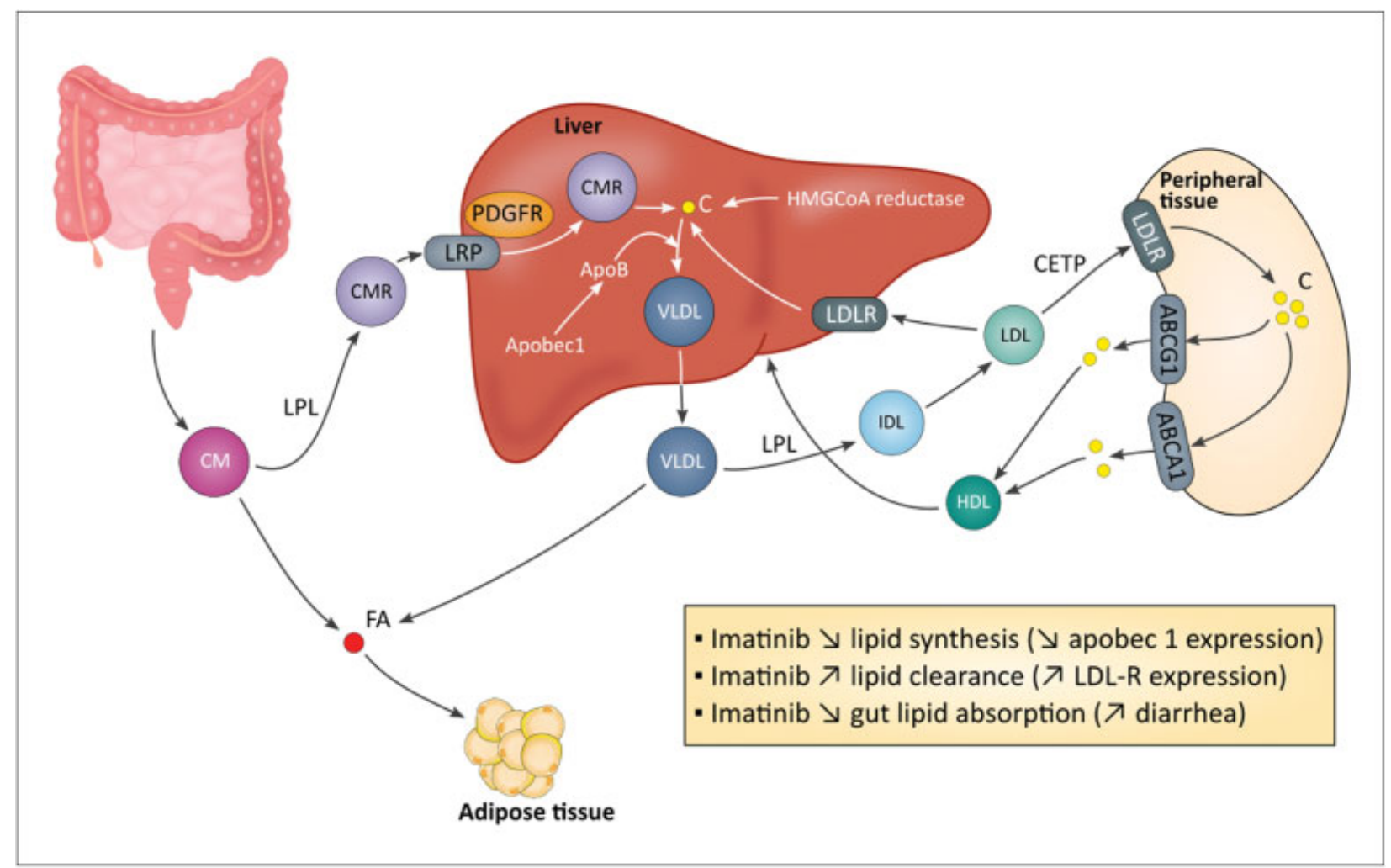

Fig. 3 Effects of BCR-ABL TKIs on lipid metabolism. Several hypotheses have been emitted to explain the imatinib-induced hypolipidemic effect. Imatinib regulates expression of genes involved in lipid metabolism: Apobec1 that regulates ApoB expression through the introduction of a stop codon into ApoB mRNA (ApoB is essential for VLDL production), and LDLR that is implicated in lipid clearance. Imatinib-induced PDGFR inhibition influences LPL synthesis and dysregulates LRP. Dasatinib and nilotinib increase cholesterol plasma level through an unknown mechanism. Global hypotheses can be emitted and include increased hepatic lipid synthesis (possibly related to hyperinsulinemia) and decreased lipid clearance through LDLR functional defect or decreased LPL synthesis. ABC, ATP-binding cassette; C, cholesterol; CETP, cholesteryl ester transfer protein; CM, chylomicron; FA, fatty acid; HMGCoA reductase, hydroxymethylglutaryl-CoA reductase; IDL, intermediate-density lipoprotein; LDL, lowdensity lipoprotein; LDLR, low-density lipoprotein receptor; LPL, lipoprotein lipase; LRP, lipoprotein receptor-related protein; PDGFR, plateletderived growth factor receptor; VLDL, very low-density lipoprotein.

Oppositely to imatinib and dasatinib, case reports and clinical trials indicate that nilotinib increases blood glucose level and promotes diabetes mellitus. ${ }^{62-65}$ Indeed, $20 \%$ of nilotinib-treated patients developed diabetes after 3 years of treatment, ${ }^{65}$ whereas $29 \%$ of patients suffer from increase of fasting glucose after 1 year of therapy. ${ }^{64}$ However, no variations of glycated hemoglobin were reported. ${ }^{64,65}$ Clinical data indicate no direct effect of nilotinib on $\beta$-cells, but suggest fasting insulin increase, fasting C-peptide decrease, and an increase of HOMA-IR values (i.e., a model to assess insulin resistance). ${ }^{64,66,67}$ Therefore, the preferred hypothesis to explain the development of hyperglycemia is the manifestation of insulin resistance. Weakened insulin secretion occurred sometimes, but it is likely that this impairment is the consequence of $\beta$-cell exhaustion. ${ }^{68}$ However, in vitro experiments demonstrated inhibitory effect of nilotinib on pancreatic cell growth. ${ }^{69}$ Breccia et al proposed an additional hypothesis linking development of hyperglycemia and body mass index. They suggested that the development of hyperglycemia might be the consequence of increase fat level tissue resulting in decrease peripheral insulin sensitivity. ${ }^{70}$ However, dietetic measures to restrict glucose exogenous uptake in patients who developed hyperglycemia were not successful, ${ }^{63}$ and nilotinib does not induce changes in patient body weight. ${ }^{71}$ Little is known regarding the mechanism by which nilotinib induces insulin resistance. Racil et al suggested that peripheral insulin resistance is mediated by c-Abl inhibition which is involved in insulin receptor signaling (-Fig. 2). ${ }^{67}$ This hypothesis is contrary to the hypothesis described with dasatinib and imatinib in which c-Abl enhances insulin sensitivity through c-Abl inhibition. These two hypotheses describe different pathways involving c-Abl but with opposite outcomes. To date, no hypothesis is preferred and additional studies are required to understand the opposite effect on glucose metabolism between TKIs, whereas both have been attributed to c-Abl inhibition. Interestingly, Frasca et al described opposite role of $\mathrm{c}-\mathrm{Abl}$ in insulin signaling depending on the receptor involved, the signaling pathway, and the cell context. ${ }^{72}$ Similar investigations should be performed in the context of $c-A b l$ inhibition by BCR-ABL TKIs. For bosutinib and ponatinib, little is known regarding their impact on glucose metabolism, but no drastic changes in glucose profile has been reported during clinical trials. 


\section{Lipid Metabolism}

Similarly with glucose metabolism, effects on lipid metabolism are conflicting between TKIs. Oppositely to in vivo study which demonstrated no impact of imatinib on total cholesterol and triglycerides levels in diabetic mice, ${ }^{29}$ imatinib is associated in CML patients with a rapid and progressive decrease of cholesterol and triglycerides levels. ${ }^{66,73-75}$ First hypothesis relates the inhibition of PDGFR by imatinib (-Fig. 3). PDGFR is involved in the synthesis of the lipoprotein lipase (LPL) and in the regulation of the lipoprotein receptor-related protein (LRP). ${ }^{73,74}$ However, all BCR-ABL TKIs possess inhibitory activity against PDGFR but do not share this positive impact on lipid profile. Recently, Ellis et al described that imatinib impairs gene expression of proteins involved in plasma lipid regulation. Indeed, in in vitro model of CML cells, imatinib affects gene expression of four genes implicated in lipid synthesis (HMG-CoA reductase ${ }^{g}$ gene and apobec $1^{\mathrm{h}}$ ), lipid clearance (LDLR gene ${ }^{\mathrm{i}}$ ) and in exchange of lipids from very low-density lipoprotein (VLDL) or lowdensity lipoprotein (LDL) to high-density lipoprotein (HDL) (CETP ${ }^{\mathrm{j}}$ gene). However, these studies were performed in a model of CML cells and need to be confirmed in more relevant models (e.g., primary cell lines, hepatocytes). ${ }^{76}$ Franceschino et al suggested that imatinib decreases diarrhea-related lipid absorption due to inhibition of c-kit in interstitial Cajal cells (i.e., c-kit signaling is critical for the survival and development of these cells). ${ }^{73}$ However, this hypothesis is unlikely, few patients (3.3\%) developed grade $3 / 4$ diarrhea, and patients treated with interferon- $\alpha$ and cytarabine developed diarrhea at a same rate and do not present lipid level reduction in the phase 3 clinical trial (NCT00333840).

Oppositely, dasatinib and mostly nilotinib are associated with an increase of cholesterol level. ${ }^{26,66,77}$ Nilotinib induces quick rise of total cholesterol, HDL, and LDL (i.e., within 3 months). Nilotinib-induced dyslipidemia are responsive to statin and lipid level normalized after nilotinib discontinuation. $^{78}$ To date, the mechanism by which dasatinib and nilotinib impact lipid metabolism is unknown. Future researches should determine how these treatments induce dyslipidemia. Global hypotheses could be formulated and include an increase of lipid synthesis that might be secondary to insulin resistance and hyperinsulinemia. This hypothesis is particularly relevant with nilotinib and it is also associated with hyperglycemia. Dasatinib and nilotinib might also decrease blood lipid clearance (e.g., disturbance of LDLR and LPL synthesis). The development of dyslipidemia might contribute to the occurrence of arterial occlusive events that occurred with nilotinib and dasatinib.

\footnotetext{
${ }^{g}$ HMGCoA reductase: 3-hydroxy-3-methyl-glutaryl-coenzyme A reductase. $H M G C O A$ reductase catalysis is the conversion of HMG-CoA to mevalonic acid, an essential step in cholesterol synthesis.

h Apobec1: Apolipoprotein B mRNA editing enzyme catalytic subunit 1. Apobec1 introduces a stop codon into ApoB mRNA.

i LDLR: Low-density lipoprotein receptor. This cell surface receptor mediates LDL endocytosis.

j CETP: Cholesteryl ester transfer protein.
}

However, the relationship between impaired lipid metabolism and cardiovascular occlusive events is unknown with BCR-ABL TKIs, and there is no indication that correct management of lipid metabolism can prevent arterial thrombosis (e.g., stenosis occurred in a nilotinib-treated patient despite the management of its hyperlipidemia through statin treatment). ${ }^{79}$ On their side, bosutinib and ponatinib do not disturb lipid metabolism. ${ }^{78,80}$

\section{Effects on Atherosclerosis}

\section{Endothelial Dysfunction}

-Fig. $\mathbf{S 1}$ in the Supplementary Material details the role of endothelial cells (ECs) in atherosclerosis. Several in vitro and in vivo experiments assess the impact of imatinib on EC viability and functions (-Table 2). These studies demonstrate that imatinib does not affect EC viability nor induce apoptosis but increases EC proliferation. ${ }^{39,81-84}$ Only one study reports a proapoptotic effect of imatinib on ECs, but their experiments were performed on a cell line (i.e., EA. hy926 cells), ${ }^{85}$ a model less reliable than primary cultures (e.g., HUVEC, ${ }^{k}$ HCAEC $^{\mathrm{l}}$ ). In vitro studies also assessed the effect of imatinib on EC functions. In these studies, imatinib does not influence adhesion molecule expressions (i.e., ICAM- $1^{\mathrm{m}}$ and VCAM- $\left.1^{\mathrm{n}}\right)$, EC migration, reactive oxygen species (ROS) production, nor angiogenesis..$^{81,82,85-87}$ Letsiou et al suggested that imatinib decreases EC inflammation by decreasing the secretion of proinflammatory mediators. ${ }^{86}$ The impact of imatinib on endothelial permeability is not clear. Indeed, in vitro studies demonstrate that imatinib increases endothelial permeability by decreasing the level of plasma membrane VE-cadherin, ${ }^{\text {o, } 85,86}$ whereas in vivo experiments indicate decreased vascular leak following imatinib treatment in a murine model of acute lung injury. ${ }^{88}$ Additionally, imatinib has been tested in patients suffering from acute lung injury, a disease characterized by vascular leakage, and demonstrate promising clinical efficacy. Therefore, imatinib might positively affect atherogenesis by decreasing endothelial inflammation and reducing vascular leakage.

Nilotinib and ponatinib reduce EC proliferation and might impaired endothelial regeneration. ${ }^{39,82,89,90}$ Additionally, ponatinib induces EC apoptosis, although it is well recognized that high glucose concentration induces EC death, ${ }^{91}$ suggesting that nilotinib might, by this intermediary, affect EC viability. Moreover, clinical data indicate that dasatinib induces pulmonary arterial hypertension, whereas imatinib is possibly beneficial in this disease. ${ }^{92,93}$ This pathology is initiated by dysfunction or injury of pulmonary ECs. ${ }^{87}$

\footnotetext{
$\overline{\mathrm{k}}$ HUVEC: Human umbilical vein endothelial cells.

1 HCAEC: Human coronary artery endothelial cells.

m ICAM-1: Intercellular adhesion molecule 1. ICAM-1 stabilizes leukocyte-endothelial cell adhesion and facilitates leukocyte transmigration.

n VCAM-1: Vascular cell adhesion molecule 1. VCAM-1 mediates rolling-type and firm adhesion of leukocyte.

o VE-cadherin: Vascular endothelial cadherin. VE-cadherin is a cellcell adhesion molecules and implies in endothelial junctions.
} 


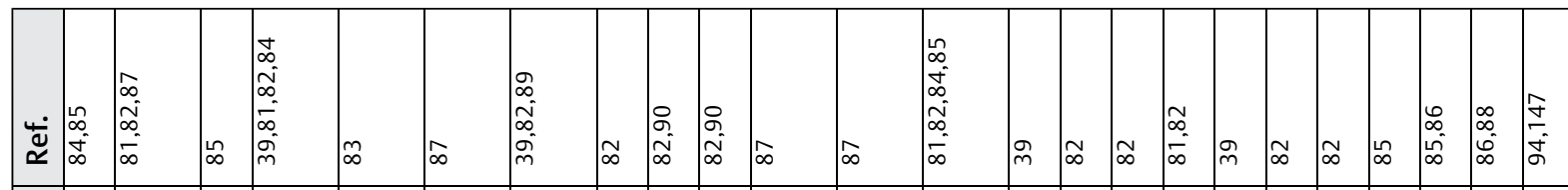

\begin{tabular}{|c|c|c|c|c|c|c|c|c|c|c|c|c|c|c|c|c|c|c|c|c|c|c|}
\hline & & & & & & & & & & & & & & 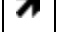 & & & 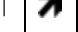 & & 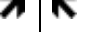 & & & \\
\hline$\frac{n}{\underline{\underline{v}}}$ & 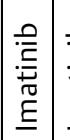 & 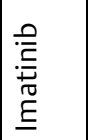 & 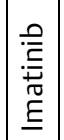 & 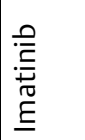 & 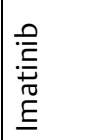 & 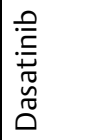 & 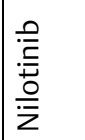 & 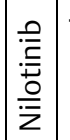 & 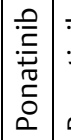 & 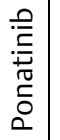 & 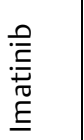 & 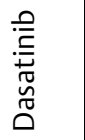 & 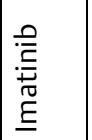 & 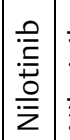 & 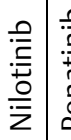 & 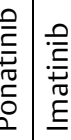 & 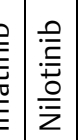 & 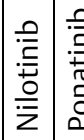 & 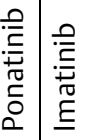 & 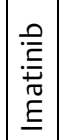 & 哭 & \\
\hline 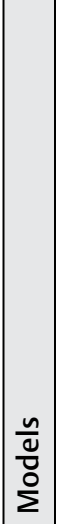 & 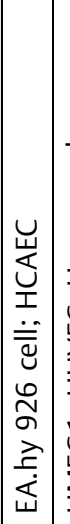 & 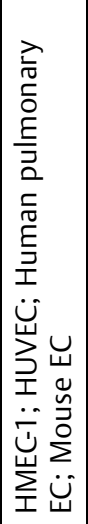 & 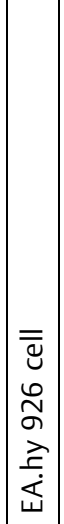 & 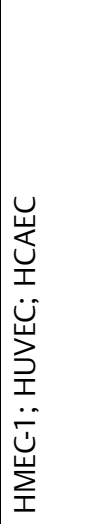 & 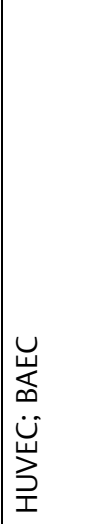 & 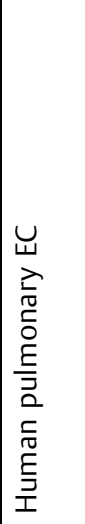 & 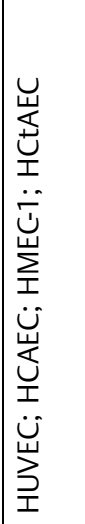 & $\sum_{\breve{Y}}^{\longrightarrow}$ & 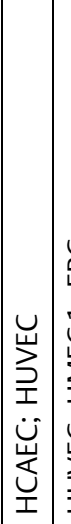 & 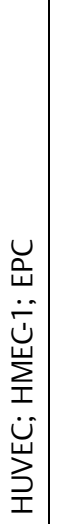 & 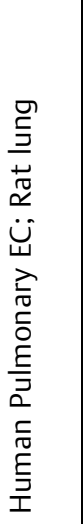 & 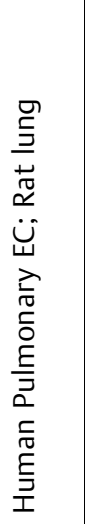 & 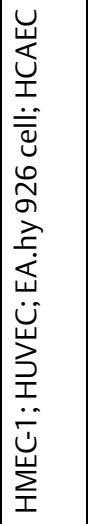 & 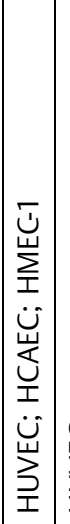 & 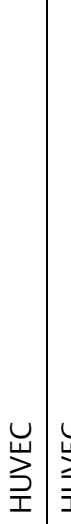 & 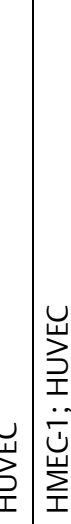 & 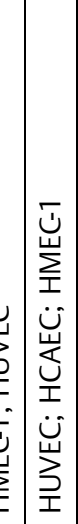 & 怠 & 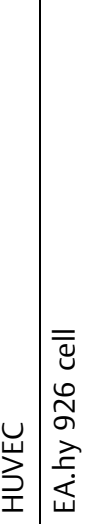 & 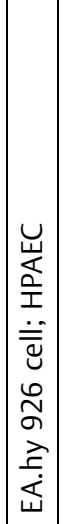 & 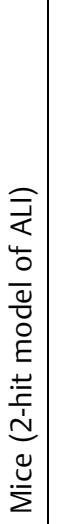 & \\
\hline 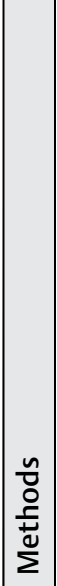 & 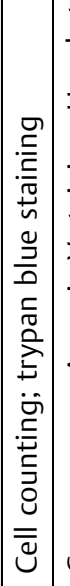 & 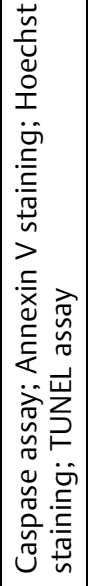 & 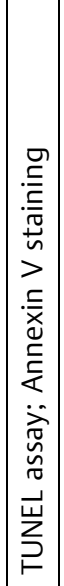 & 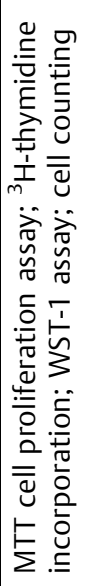 & 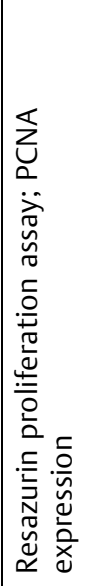 & 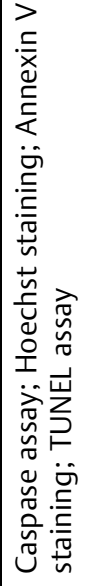 & 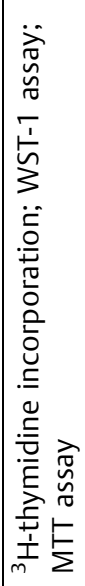 & 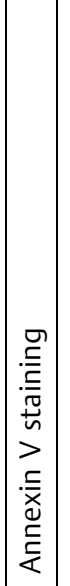 & 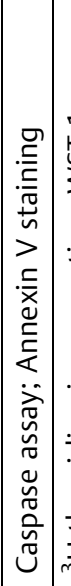 & 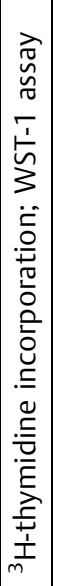 & 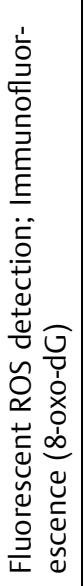 & 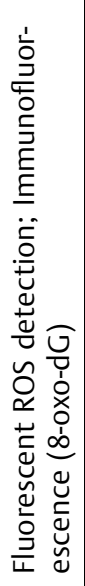 & 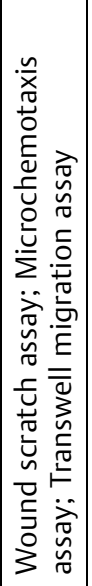 & 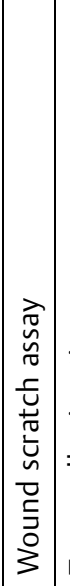 & 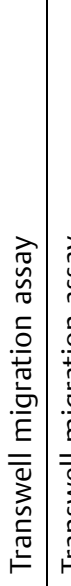 & 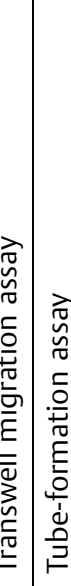 & 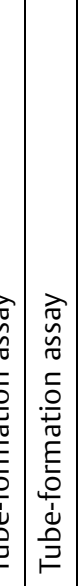 & 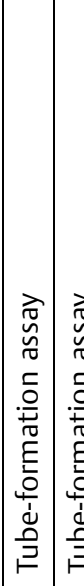 & 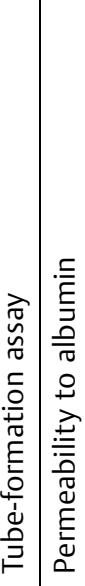 & 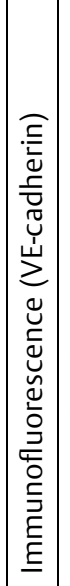 & 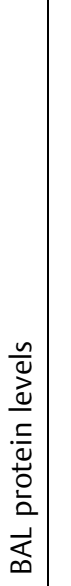 & \\
\hline 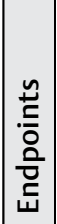 & 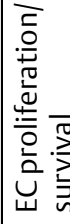 & & & & & & & & & & 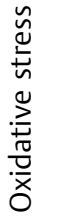 & & 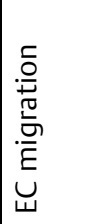 & & & 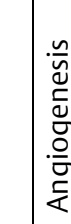 & $\begin{array}{l}\frac{\hat{n}}{\bar{y}} \\
\frac{5}{2} \\
\frac{5}{2}\end{array}$ & & 竞 & & & \\
\hline
\end{tabular}




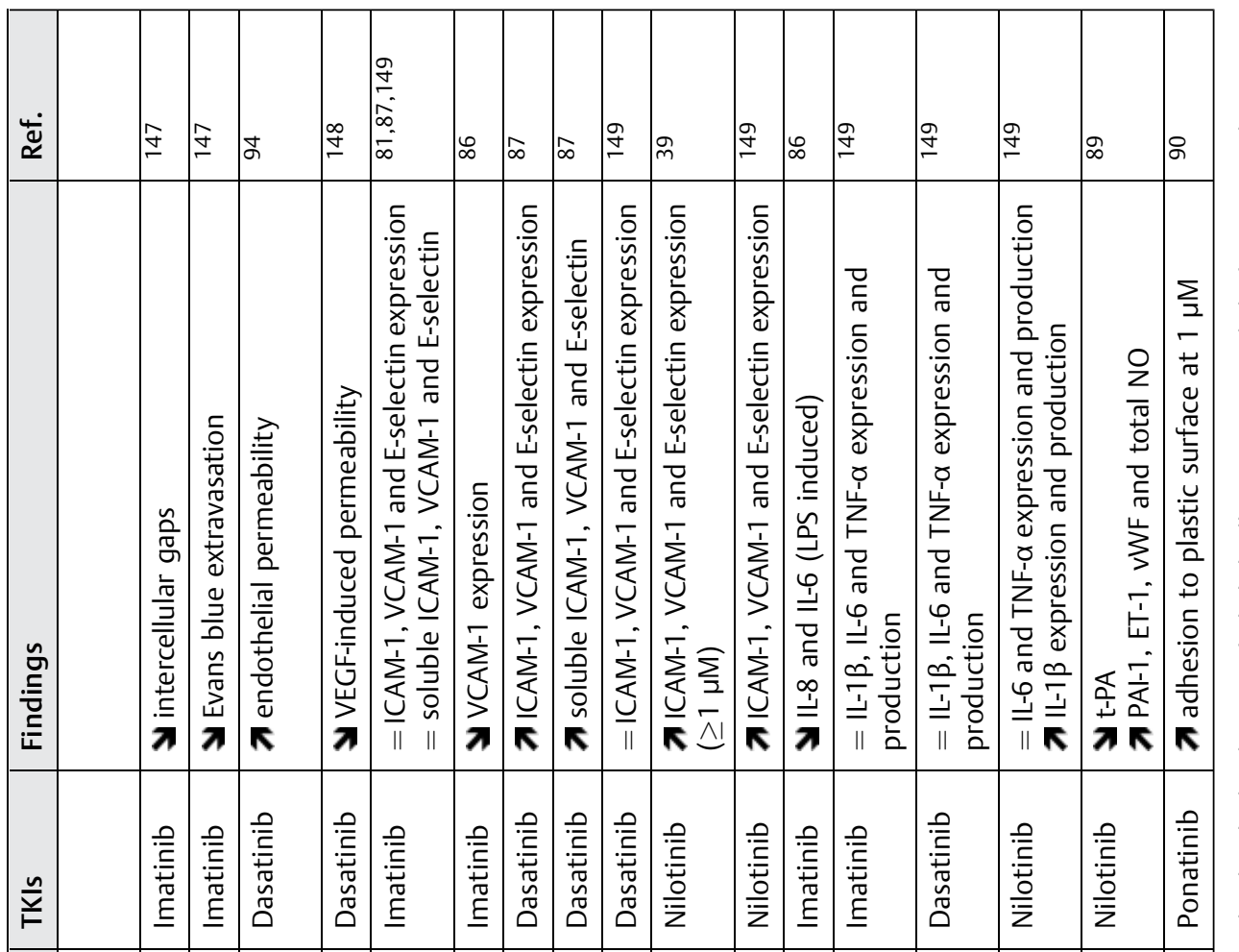

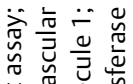

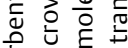
政

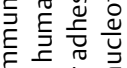
它空 就 突言.

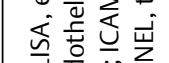

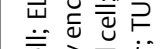

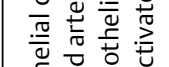

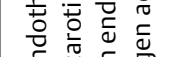
过

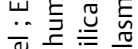

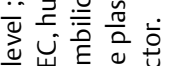

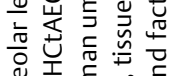

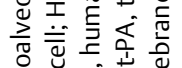

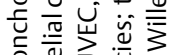

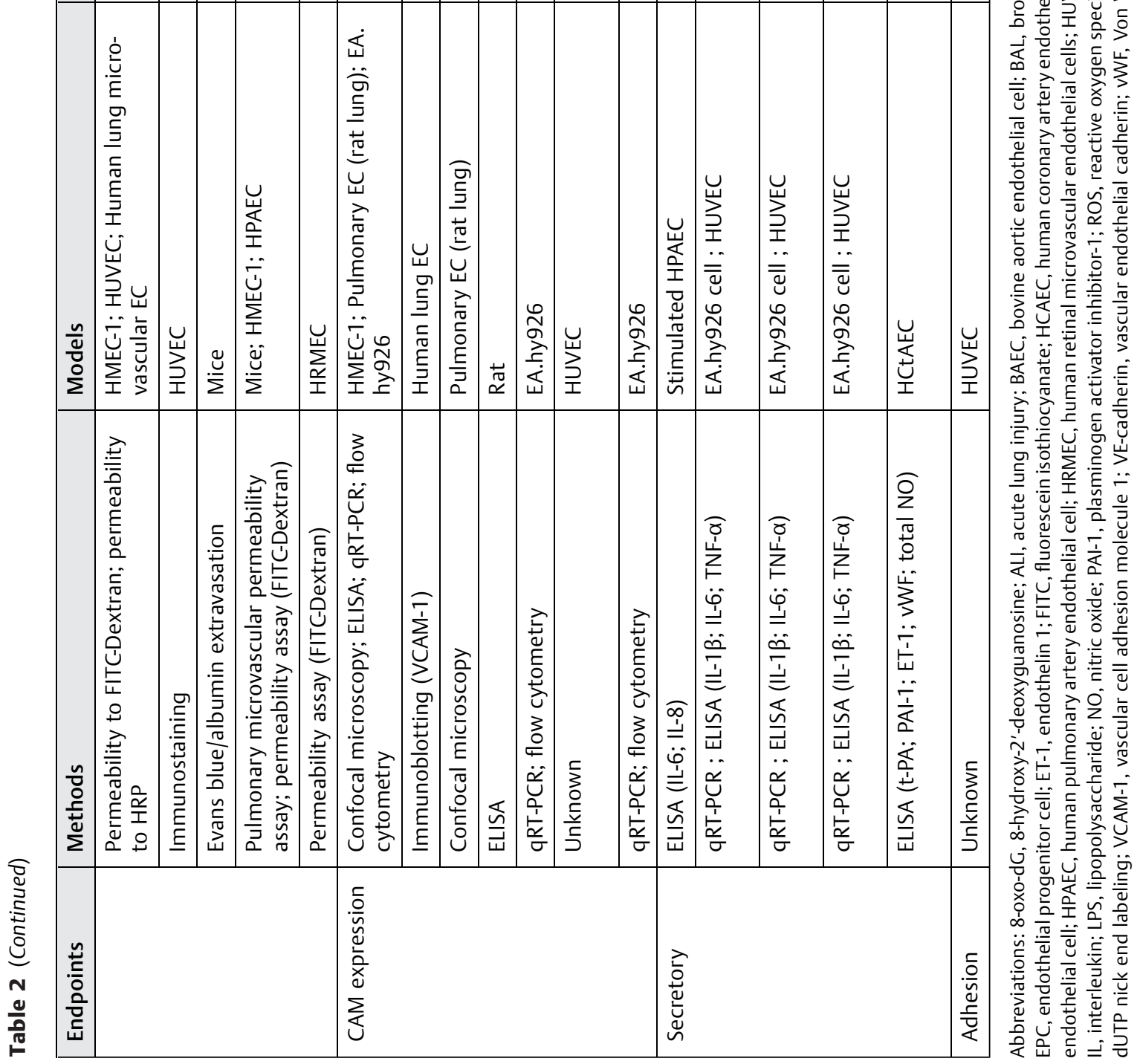


Therefore, in vivo and in vitro studies investigated effect of imatinib and dasatinib on pulmonary ECs and demonstrate that dasatinib induces apoptosis on pulmonary ECs mediated by increased mitochondrial ROS production. ${ }^{87}$ Future researches should assess if this effect is also found in arterial ECs and ROS production should also be tested with other new-generation BCR-ABL TKIs.

In addition to their effect on EC viability, nilotinib and ponatinib also influence EC functions, inhibit their migration, and decrease angiogenesis. ${ }^{39,82}$ It was suggested that the antiangiogenic effect of ponatinib is the consequence of VEGFR $^{\mathrm{p}}$ inhibition, but this hypothesis cannot explain the antiangiogenic effect of nilotinib (i.e., nilotinib does not inhibit VEGFR). ${ }^{82}$ Nilotinib also increases adhesion molecule expressions (i.e., ICAM-1, VCAM-1, and E-selectin) in vitro, ${ }^{39}$ suggesting that nilotinib might increase leukocyte recruitment. However, further experiments are needed to validate this hypothesis (e.g., assessment of endothelium permeability and transendothelial migration). Dasatinib also induces endothelium leakage in vitro, and the RhoA-ROCK ${ }^{\mathrm{q}}$ pathway is involved in this phenomenon. ${ }^{94}$ It was demonstrated that RhoA activation induces the phosphorylation of myosin light chain that increases the actomyosin contractibility and disrupt endothelial barrier. ${ }^{94}$ Therefore, increased endothelium permeability is a potential mechanism by which dasatinib and nilotinib promote atherosclerosis development and arterial thrombosis. Likewise, it is plausible that ponatinib affects endothelium integrity because of its inhibitory activity against VEGFR, which is recognized as a permeabilityinducing agent. Additional hypotheses suggest that inhibition of Abl kinase (i.e., $\operatorname{Arg}^{\mathrm{r}}$ and c-Abl) and PDGFR might also be implicated in vascular leakage. ${ }^{85}$ Finally, Guignabert et al demonstrated that both in rats and in CML patients taking dasatinib, there is an increase of soluble adhesion molecules, which are well-known markers of endothelial dysfunction. ${ }^{87}$

\section{Inflammation}

-Fig. S2 in the Supplementary Material describes the role of immune cells and inflammation process during atherosclerosis. - Table 3 summarizes in vitro studies that investigate impacts of BCR-ABL TKIs on survival, proliferation, and major functions of monocytes, macrophages, and Tlymphocytes. Globally, in vitro studies demonstrate that imatinib inhibits the development and maturation of monocytes and alters monocyte functions. ${ }^{95,96}$ Imatinib decreases production of proinflammatory cytokines (i.e., TNF- $\alpha^{\mathrm{s}}$ and IL- ${ }^{\mathrm{t}}$ ) and diminishes the potential of monocytes to phago-

p VEGFR: Vascular endothelial growth factor. This protein plays major roles in vasculogenesis and angiogenesis.

q RhoA-ROCK: Ras homolog gene family, member A-Rho-associated protein kinase. Rho-kinase regulates cytoskeletal reorganization, cell migration, cell proliferation, and survival.

$r$ Arg: Abelson-related gene (also known as ABL2). Arg possesses cytoskeletal-remodeling functions.

$s$ TNF- $\alpha$ : Tumor necrosis factor alpha. This cytokine is mainly involved in systemic inflammation and regulates immune cells.

t IL-6: Interleukin 6. IL-6 is a proinflammatory cytokine secreted by $T$-cells and macrophages to stimulate immune response. cytose. ${ }^{97,98}$ These impacts on monocyte functions are possibly related to $\mathrm{c}-\mathrm{fms}^{\mathrm{u}}{ }^{\mathrm{inh}}$ ibition. ${ }^{99}$ Imatinib also inhibits macrophage functions in vitro. Imatinib decreases lipid uptake without impacting the lipid efflux and decreases activity and secretion of two matrix metalloproteinases (MMPs; i.e., MMP-2 and MMP-9v ${ }^{\mathrm{v}}$ ) on a posttranscriptional level. ${ }^{100}$ Additionally, imatinib inhibits T-lymphocyte activation and proliferation and decreases proinflammatory cytokines secretion (i.e., IFN- $\gamma^{w}$ ). ${ }^{101}$ The inhibition of monocyte, macrophage, and T-cell functions by imatinib might prevent the development of atherosclerosis or reduce the risk of atherosclerotic plaque rupture.

Effects of new-generation TKIs on inflammatory cells were less studied, but first experiments indicate similarities with imatinib about its impact on monocytes and macrophages. Both dasatinib and nilotinib have similar inhibitory profile on macrophage-colony formation that has been linked to CSFR inhibition. ${ }^{96,102}$ Dasatinib also possesses anti-inflammatory functions by attenuating proinflammatory cytokines production (i.e., TNF- $\alpha$, IL- 6 , and IL- $12^{\mathrm{x}}$ ) by macrophages and increasing production of anti-inflammatory mediator (i.e., IL-10 ${ }^{\mathrm{y}}$ ). ${ }^{103}$ These effects are thought to be mediated by $\mathrm{SIK}^{\mathrm{z}}$ inhibition, a subfamily of three serine/ threonine kinases that regulate macrophage polarization. ${ }^{103,104}$ Finally, dasatinib is associated with decreased T-cell functions and particularly it decreases the production of proinflammatory cytokines (e.g., TNF- $\alpha$, IFN- $\gamma$ ) and chemotactic mediators. ${ }^{105}$ Nilotinib and bosutinib also possess anti-inflammatory activity and decrease cytokine production and T-cell activation. ${ }^{103,106}$ Inhibition of Lck, ${ }^{\text {ai }}$ a tyrosine kinase implicated in T-cell receptor signaling, is implicated in the impairment of T-cell functions by dasatinib and nilotinib. ${ }^{107,108}$ It has been hypothesized that nilotinib decreases mast cell activity through c-kit inhibition, ${ }^{62,109}$ which might result in a decrease of the vascular repair system. ${ }^{39,62}$ Clinical profile of nilotinib in patients with CML consolidates this hypothesis and demonstrates a decreased of mast cell level. ${ }^{39}$ However, similar decreased of mast cell is also reported with imatinib without high rate of arterial thrombosis. $^{110}$

Globally, BCR-ABL TKIs possess reassuring profile on inflammatory cells. However, impact of new-generation TKIs on several functions of macrophages have not been assessed (e.g., MMP secretion and activity, lipid uptake, and

\footnotetext{
u CSFR: Colony-stimulating factor receptor. CSFR drives growth and development of monocytes.

v MMP-2 and MMP-9 are two proteases capable of degrading extracellular matrix components. These two MMPs are the main proteases involved in atherogenesis.

${ }^{\mathrm{w}}$ IFN- $\gamma$ : Interferon gamma. IFN- $\gamma$ is involved in innate and adaptive immunity and activates macrophages.

x IL-12: Interleukin 12. IL-12 is involved in T-cell differentiation and functions.

y IL-10: Interleukin 10. IL-10 exerts immunoregulation and regulates inflammation.

z SIK: Salt-inducible kinase. SIKs regulate production of anti- and proinflammatory cytokines.

ai Lck: Lymphocyte-specific protein tyrosine kinase. Lck is mostly involved in T-cell maturation.
} 


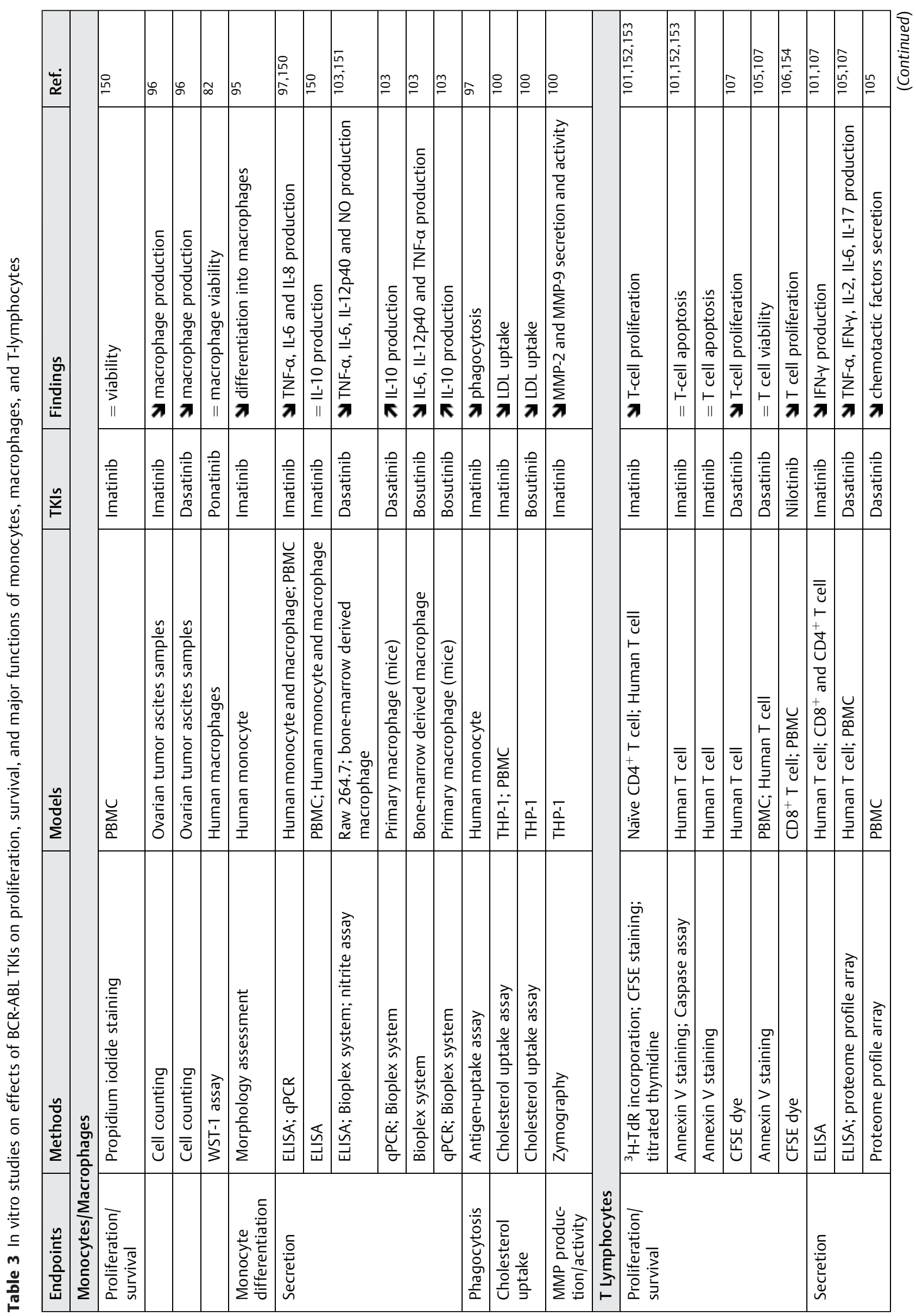




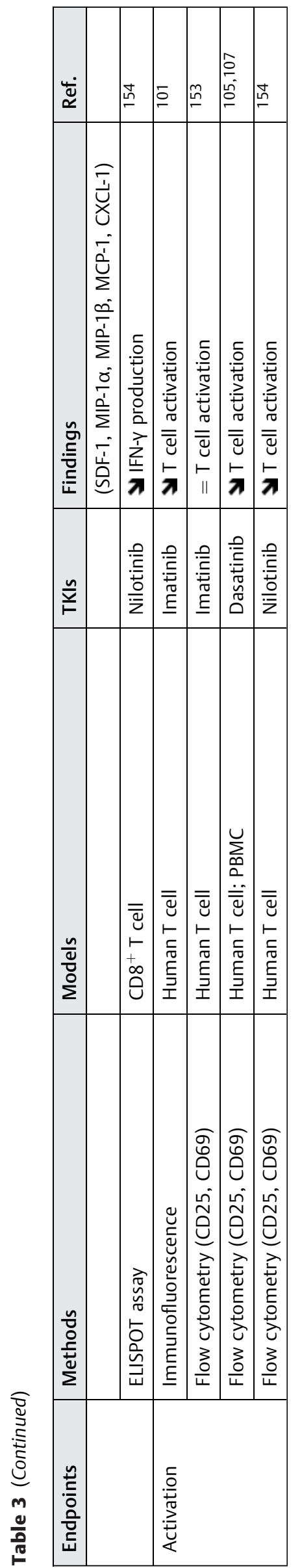

foam cell formation), whereas effect of ponatinib on inflammatory cells is unknown. The assessment of lipid uptake and foam cell formation is particularly relevant with new-generation TKIs because there are numerous interactions between TKIs and ABC transporters. ${ }^{\text {aii, } 111,112}$

\section{Fibrous Cap Thickness}

- Fig. $\mathbf{5 3}$ in the Supplementary Material describes the mechanism by which atherosclerotic plaque ruptures and induces arterial thrombosis. - Table 4 summarizes in vitro and in vivo experiments performed on VSMCs and fibroblasts. Imatinib decreases VSMC proliferation and growth but results are conflicting about its impact on apoptosis. Some studies demonstrate no impact on SMC apoptosis, whereas others indicate increased SMC death. ${ }^{83,113-116}$ Imatinib also affects VSMC functions and decreases their migration and LDL binding, inducing decreased LDL retention by the sub-endothelium. ${ }^{113,117}$ Imatinib also exerts negative effect on the synthesis of major ECM components (type I collagen and fibronectin A) by fibroblasts, correlating to decreased ECM accumulation in vivo. ${ }^{118}$ The impact of imatinib on SMCs is thought to be mediated by PDGFR inhibition, ${ }^{114}$ which is involved in several VSMC functions including VSMC survival and plasticity. ${ }^{113}$ Subsequent to the hypothesis that imatinib inhibits PDGFR signaling, prevents abundant SMC and fibroblast proliferation, and inhibits abundant ECM accumulation, imatinib has been tested for the management of several fibrotic diseases (e.g., dermal and liver pulmonary fibrosis, systemic sclerosis). ${ }^{30,118,119}$ Imatinib successfully acts on pulmonary fibrosis and pulmonary arterial hypertension (i.e., a disease involving vascular remodeling mediated by pulmonary SMC proliferation), ${ }^{93,114}$ and has beneficial activity in sclerotic chronic graft-versus-host disease. ${ }^{120}$ Finally, imatinib was tested in vivo for the prevention of cardiovascular diseases and demonstrates efficacy for the treatment of myocardial fibrosis by reducing ECM component synthesis (i.e., procollagen I and III). ${ }^{30}$ In a rat model, imatinib successfully inhibits stenosis after balloon injury and presents interest in intimal hyperplasia and stenosis after bypass grafts. ${ }^{115,116,121-123}$ Imatinib also successfully prevents arterial thrombosis following microvascular surgery in rabbits. ${ }^{124}$ Imatinib was also encompassed in a stent but do not demonstrate efficacy in restenosis prevention. ${ }^{84}$

Impact of new-generation TKIs on fibrosis was less studied but demonstrate similar inhibitory effect on VSMCs and fibroblasts. Indeed, dasatinib inhibits PDGFR more potently than imatinib, ${ }^{113}$ and the hypothesis that dasatinib prevents restenosis similarly with imatinib was emitted. Therefore, a patent has been filed claiming the use of dasatinib for the prevention of stenosis and restenosis. ${ }^{125}$ Compared with imatinib, dasatinib has additional off-targets and is able to

\footnotetext{
${ }^{a i i} A B C$ transporter: ATP-binding cassette transporters. ABCG1 and $A B C A 1$ are implicated in macrophage reverse cholesterol transport. Their deficiency leads to foam cell formation and atherosclerosis development.
} 


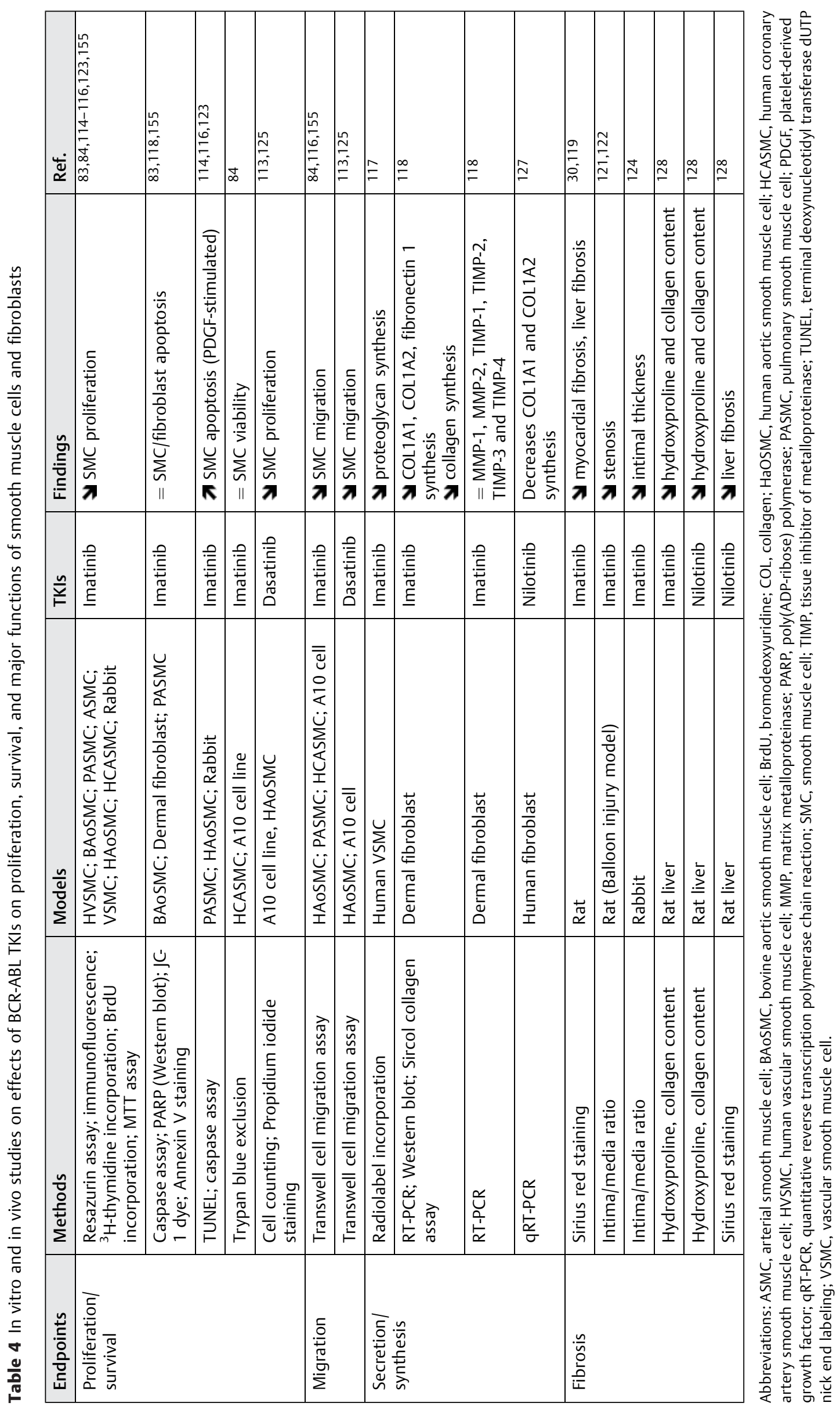




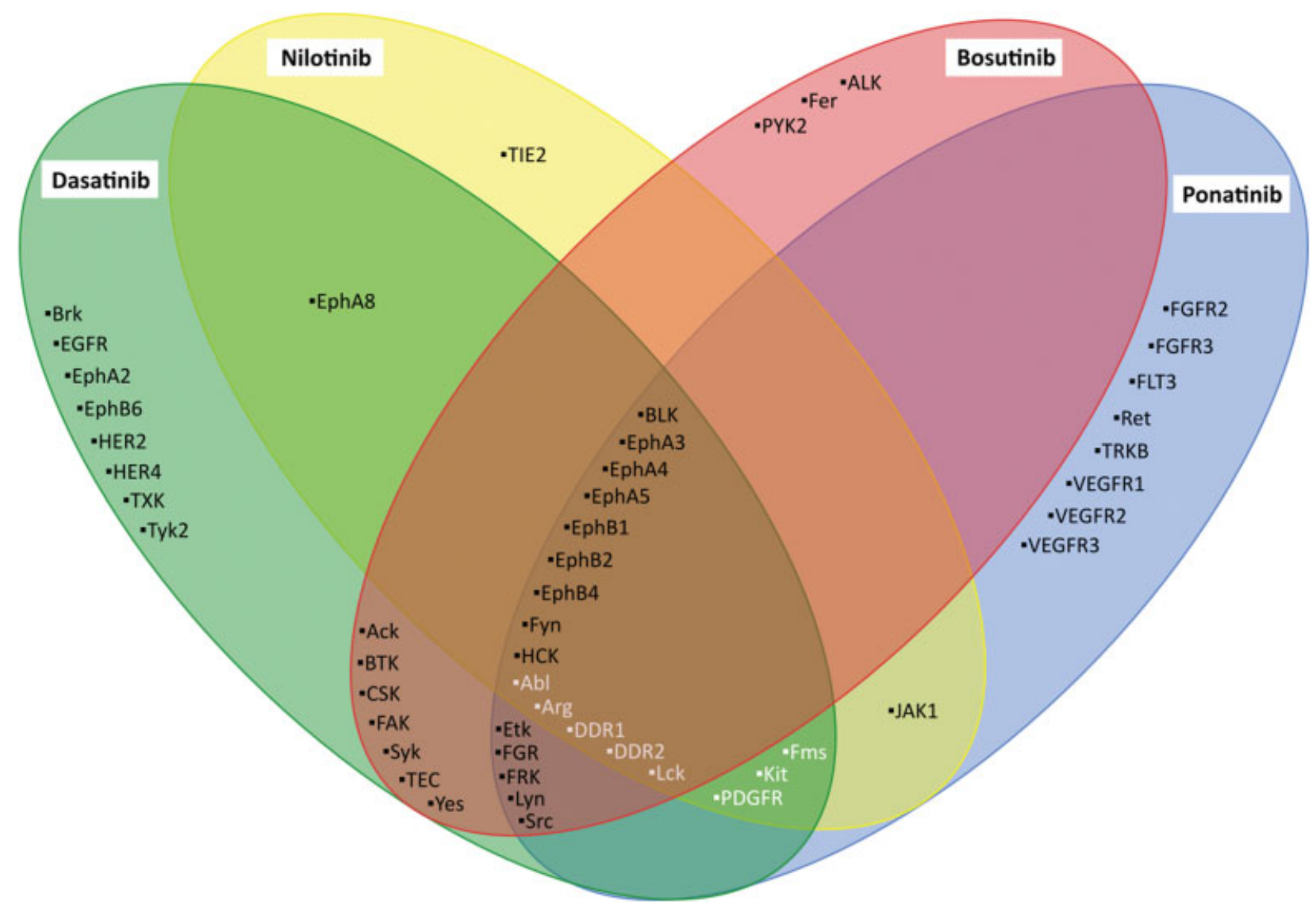

Fig. 4 Specificity of imatinib, dasatinib, nilotinib, and ponatinib toward tyrosine kinases. Green, yellow, red, and blue circles contain tyrosine kinase inhibited by dasatinib, nilotinib, bosutinib, and ponatinib, respectively. Tyrosine kinases in white represent imatinib off-targets. This figure summarizes results from 13 experiments. ${ }^{39,43,130,132-137,156-159}$ In case of conflictual results between studies, a conservative approach has been applied. Additional information is provided in the Supplementary Material.

inhibit Src, aiii a kinase involved in dermal fibrosis in addition to PDGFR. ${ }^{126}$ Therefore, dasatinib was tested in patients with scleroderma-like chronic graft-versus-host disease, a disease resulting from inflammation and progressive fibrosis of the dermis and subcutaneous tissues, and first results are encouraging. ${ }^{126}$ Nilotinib also appears to be clinically efficient in scleroderma-like graft-versus-host disease by reducing collagen expression. ${ }^{127}$ Finally, nilotinib was tested in vivo for the treatment of liver fibrosis and demonstrates decreased fibrotic markers and inflammatory cytokines (IL$1 \alpha$, IL- $1 \beta$, IFN- $\gamma$, IL-6). ${ }^{128}$ However, only low-dose nilotinib was found to be efficient against fibrosis and normalized collagen content. ${ }^{128}$ This lack of antifibrotic effect at higher doses might be explained by inhibition of additional offtargets by nilotinib that affect the benefit of low-dose nilotinib against fibrosis. Arterial thrombosis occurring with dasatinib and nilotinib are probably not the consequence of VSMC impairment, but investigations should be performed on VSMCs rather than on fibroblasts. Additional investigations are warranted to complete impact of BCR-ABL TKIs on VSMC functions (e.g., VSMC apoptosis, proliferation, and migration) and confirm their safety toward VSMCs.

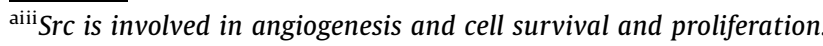

\section{Off-targets}

BCR-ABL TKIs bind the highly conserved ATP binding site and are therefore not very specific to BCR-ABL and possess multiple cellular targets (kinases and nonkinase proteins). ${ }^{129,130}$ This allowed the possibility to exploit them in other indications (e.g., PDGFR inhibition by imatinib is used in BCR-ABL-negative chronic myeloid disorders), ${ }^{131}$ but this may also induce toxicities and side effects. ${ }^{129}$ The development of arterial thrombotic events with new-generation BCR-ABL TKIs is likely to be related to inhibition of off-targets, as described throughout this review. - Fig. 4 describes inhibitory profiles of imatinib, dasatinib, nilotinib, bosutinib, and ponatinib. Globally, imatinib is the most selective BCR-ABL TKIs, whereas dasatinib and ponatinib inhibit numerous off-targets.

However, inhibitory profiles are difficult to determine and several researches published discrepancies. For conflicting results, a conservative approach has been applied in - Fig. 4, but supplementary information ( - Table S2) describes the tyrosine kinase selectivity profile of the five BCR-ABL TKIs and indicates divergences between studies. ${ }^{43,130,132-134}$ These discrepancies can be explained by the difference in drug concentration and methodologies. To date, several methods have been used to determine inhibitory profile of BCR-ABLTKIs including in vitro kinase assay, ${ }^{133-135}$ kinase expression in bacteriophages, ${ }^{136}$ and 
affinity purification methods combined with mass spectrophotometry. ${ }^{130,132}$ However, all these methods suffer from caveats, including the incompatibility to perform live-cell studies. A cellpermeable kinase probe was developed to figure out this problem, but this assay is still limited by the number of off-target tested (i.e., it requires to predefine tested off-targets) and therefore, the missing of targets is possible. ${ }^{137}$ For this reason, the inhibitory activity of each TKI has not been tested toward all tyrosine kinase and -Fig. 4 includes only off-targets for which at least one of the five BCR-ABLTKI has been tested. Thus, inhibitory profiles need to be carefully considered and it has to keep in mind that BCR-ABL TKI metabolites may possess activity against supplemental offtargets.

As described over this review, PDGF signaling has countless effects on several cells and tissues and is involved in several proatherogenic mechanisms (e.g., adipogenesis, vascular leakage, VSMC viability, and functions) and vascular homeostasis, which led to the suggestion of its implication in the potential beneficial cardiovascular effect of imatinib. ${ }^{116,123,138}$ However, dasatinib, nilotinib, and ponatinib also inhibit PDGFR but increase the risk of arterial occlusive events. This difference of clinical outcome might be explained by the concentration of BCR-ABL TKIs necessary to obtain a same degree of PDGFR inhibition. ${ }^{43}$ Indeed, Rivera et al reported that when adjusted to the maximum serum concentration, imatinib inhibits more profoundly PDGFR than dasatinib, nilotinib, and ponatinib. ${ }^{43}$ Therefore, at effective concentration, it is probable that the degree of PDGFR inhibition is too low with dasatinib, nilotinib, and ponatinib to obtain the beneficial effect of PDGFR inhibition on atherosclerosis. Another possible hypothesis concerns the less conclusive specificity of new-generation TKIs which leads to inhibition of additional off-targets that might counterbalance the positive effect of PDGFR inhibition.

Other tyrosine kinases have been incriminated in the occurrence of arterial thrombosis with new-generation TKIs. DDR- 1 aiv possesses functions in vascular homeostasis, atherogenesis, and is expressed in pancreatic islet cells. However, and similarly with PDGFR, it is inhibited by all BCR-ABL TKIs. ${ }^{26,62}$ Other hypotheses include impairment of VEGF signaling by ponatinib ${ }^{43,90}$ or the inhibition of several ephrin receptors by new-generation TKIs but not by imatinib which might inhibit monocyte recruitment. ${ }^{139}$ Finally, it has been suggested that the inhibition of c-Abl itself is implicated in the increase of the cardiovascular risk. Indeed, imatinib possesses lower inhibitory effect on c-Abl than new-generation TKIs, which might further explain the difference in cardiovascular safety. ${ }^{43}$ Additionally, c-Abl modulates Tie-2, av a tyrosine kinase that possesses impor-

\footnotetext{
$\overline{{ }^{\text {aiv }} D D R-1:}$ Discoidin domain receptor tyrosine kinase 1. DDR1 is involved in the regulation of cell growth, differentiation, and metabolism.

av Tie-2: Tunica interna endothelial cell kinase. Tie-2 regulates angiogenesis, endothelial cell survival, proliferation, migration, adhesion and cell spreading, cytoskeleton reorganization, and vascular quiescence. Tie-2 also possesses anti-inflammatory functions by preventing the leakage of proinflammatory mediators and leukocytes.
}

tant effect on endothelial cell function, angiogenesis, and inflammation. ${ }^{140,141}$

\section{Perspectives and Conclusions}

This review summarizes the data underlying the potential preventive effect of imatinib on the occurrence of arterial thrombosis. Globally, in vitro and in vivo experiments demonstrate that imatinib possesses antiplatelet activity, hypolipidemic and hypoglycemic effects, and inhibits inflammation and atherosclerosis development in several cell types (i.e., decreases of inflammatory cell and VSMC functions and increased vascular permeability). These benefits were largely attributed to PDGFR inhibition. It is currently unknown why new-generation TKIs that also inhibit PDGFR present opposite cardiovascular safety profile and this point needs to be elucidated.

New-generation BCR-ABL TKIs increase the risk of arterial thromboembolism with different clinical features (e.g., timeto-event and absolute rate) and are associated with different safety profiles, suggesting different pathways to explain the pathophysiology. The safety profile of nilotinib is mostly characterized by impaired glucose and lipid metabolism. However, both the molecular mechanism of these alterations and their impact on the occurrence of arterial thrombosis are unknown. Both dasatinib and ponatinib exhibit antiplatelet effect, whereas it was recently suggested that nilotinib potentially induces prothrombotic phenotype of platelets. Based on the clinical characteristics and case reports, atherosclerosis appears the most plausible mechanisms by which new-generation TKIs induce arterial thrombosis. However, in vitro and in vivo studies of viability and functions of SMCs and inflammatory cells demonstrate reassuring impact of dasatinib and nilotinib, even if additional studies are required to complete this evaluation. However, first experiments indicate that dasatinib, nilotinib, and ponatinib influence EC survival and/or endothelium integrity, suggesting a reasonable hypothesis by which new-generation TKIs induce atherosclerosis development and, subsequently, arterial thrombosis. Additional studies on the shedding of functional extracellular vesicles by endothelial cells might be interesting regarding their important role in coronary artery diseases. $^{142}$ Finally, the impact of new-generation TKIs on human blood coagulation and fibrinolysis has never been studied and should be addressed.

To conclude, new-generation TKIs increase the risk of arterial thrombosis in patients with CML, whereas imatinib, the first-generation TKI, might prevent the development of cardiovascular events. To date, the cellular events and signaling pathways by which these events occurred are unknown and researches are extremely limited focusing mainly on imatinib and nilotinib. Researches need to be extended to all new-generation BCR-ABL TKIs (i.e., dasatinib, bosutinib, and ponatinib). The understanding of the mechanisms by which new-generation BCR-ABL TKIs induce or promote arterial occlusive events will improve the clinical uses of these therapies. To date, only general risk minimization measures have been proposed (e.g., management of dyslipidemia, 
diabetes, arterial hypertension following standard of care). ${ }^{14,22,23,143-146}$ The understanding of the pathophysiology is required to implement the most appropriate risk minimization strategies for thrombotic events and to select patients to whom the prescription of these drugs should be avoided when applicable. Finally, the understanding of the pathophysiology will help in the design of new BCR-ABL inhibitors sparing the toxic targets.

\section{Review Criteria}

Relevant articles published from the database inception to July 11, 2017, were identified from an electronic database (PubMed) using the keywords "vascular," "thrombosis," "atherosclerosis," “arteriosclerosis," “venous," “arterial," "hemostasis," "metabolic," "metabolism," "glycemia," "glycaemia," "cholesterol," "triglycerides," and "platelet" combined with the five approved BCR-ABL TKIs. The search strategy is presented in supplementary files. Articles published in languages other than English were excluded from the analysis. Primary criteria were pathophysiological explanation of arterial thrombotic events. Abstracts and full-text articles were reviewed with a focus on atherogenesis, plaque rupture, platelet functions, and their link with the development of arterial thrombosis with BCR-ABL TKIs. The reference section of identified articles was also examined.

\section{Authors' Contributions}

H.H. was responsible for the first draft of the manuscript. F.M., C.C., C.G., J.M.D., and J.D. contributed to the final draft of the manuscript.

\section{Conflicts of Interest}

J.D. reports personal fees from Roche Diagnostics, Stago Diagnostica, Bayer Healthcare, and Daiichi-Sankyo; travel grants from Bayer Healthcare, Boehringer Ingelheim, CSL Behring, and Stago Diagnostica outside the submitted work.

F.M. reports personal fees from Boehringer Ingelheim, Bayer Healthcare, and Bristol-Myers Squibb-Pfizer outside the submitted work.

C.G. reports personal fees from Novartis, Celgene, and Amgen outside the submitted work.

The other authors have no conflicts of interest to disclose.

\section{References}

1 Bhamidipati PK, Kantarjian H, Cortes J, Cornelison AM, Jabbour E. Management of imatinib-resistant patients with chronic myeloid leukemia. Ther Adv Hematol 2013;4(02):103-117

2 Gorre ME, Mohammed M, Ellwood K, et al. Clinical resistance to STI-571 cancer therapy caused by BCR-ABL gene mutation or amplification. Science 2001;293(5531):876-880

3 Bixby D, Talpaz M. Mechanisms of resistance to tyrosine kinase inhibitors in chronic myeloid leukemia and recent therapeutic strategies to overcome resistance. Hematology (Am Soc Hematol Educ Program) 2009;2009(01):461-476

4 Cortes JE, Saglio G, Kantarjian HM, et al. Final 5-year study results of DASISION: the Dasatinib Versus Imatinib Study in TreatmentNaïve Chronic Myeloid Leukemia Patients Trial. J Clin Oncol 2016; 34(20):2333-2340
5 Hochhaus A, Saglio G, Hughes TP, et al. Long-term benefits and risks of frontline nilotinib vs imatinib for chronic myeloid leukemia in chronic phase: 5-year update of the randomized ENESTnd trial. Leukemia 2016;30(05):1044-1054

6 Brümmendorf TH, Cortes JE, de Souza CA, et al. Bosutinib versus imatinib in newly diagnosed chronic-phase chronic myeloid leukaemia: results from the 24-month follow-up of the BELA trial. Br J Haematol 2015;168(01):69-81

7 Mealing S, Barcena L, Hawkins N, et al. The relative efficacy of imatinib, dasatinib and nilotinib for newly diagnosed chronic myeloid leukemia: a systematic review and network metaanalysis. Exp Hematol Oncol 2013;2(01):5

8 Lamontanara AJ, Gencer EB, Kuzyk O, Hantschel O. Mechanisms of resistance to BCR-ABL and other kinase inhibitors. Biochim Biophys Acta 2013;1834(07):1449-1459

9 Pagnano KB, Bendit I, Boquimpani C, et al; Latin American Leukemia Net (LALNET). BCR-ABL mutations in chronic myeloid leukemia treated with tyrosine kinase inhibitors and impact on survival. Cancer Invest 2015;33(09):451-458

10 Ursan ID, Jiang R, Pickard EM, Lee TA, Ng D, Pickard AS. Emergence of BCR-ABL kinase domain mutations associated with newly diagnosed chronic myeloid leukemia: a meta-analysis of clinical trials of tyrosine kinase inhibitors. J Manag Care Spec Pharm 2015;21(02):114-122

11 National Comprehensive Cancer Network. NCCN Clinical Practice Guidelines in Oncology: Chronic Myeloid Leukemia. Version 1.2017. 2016

12 Thanopoulou E, Judson I. The safety profile of imatinib in CML and GIST: long-term considerations. Arch Toxicol 2012;86(01):1-12

13 Kalmanti L, Saussele S, Lauseker M, et al. Safety and efficacy of imatinib in CML over a period of 10 years: data from the randomized CML-study IV. Leukemia 2015;29(05):1123-1132

14 Moslehi JJ, Deininger M. Tyrosine kinase inhibitor-associated cardiovascular toxicity in chronic myeloid leukemia. J Clin Oncol 2015;33(35):4210-4218

15 Breccia M, Alimena G. Pleural/pericardic effusions during dasatinib treatment: incidence, management and risk factors associated to their development. Expert Opin Drug Saf 2010;9(05): 713-721

16 European Medicines Agency. Sprycel - Summary of Product Characteristics 2017. Available at: http://www.ema.europa.eu/docs/en _GB/document_library/EPAR_-_Product_Information/human/000 709/WC500056998.pdf. Accessed December 22, 2017

17 European Medicines Agency. Iclusig - Summary of Product Characteristics 2017. Available at: http://www.ema.europa. eu/docs/en_GB/document_library/EPAR_-_Product_Information/human/002695/WC500145646.pdf. Accessed December 22, 2017

18 European Medicines Agency. Tasigna - Summary of Product Characteristics 2017. Available at: http://www.ema.europa.eu/docs/en _GB/document_library/EPAR_-_Product_Information/human/000 798/WC500034394.pdf. Accessed December 22, 2017

19 Kantarjian HM, Cortes JE, Kim DW, et al. Bosutinib safety and management of toxicity in leukemia patients with resistance or intolerance to imatinib and other tyrosine kinase inhibitors. Blood 2014;123(09):1309-1318

20 Saglio G, Kim D-W, Issaragrisil S, et al; ENESTnd Investigators. Nilotinib versus imatinib for newly diagnosed chronic myeloid leukemia. N Engl J Med 2010;362(24):2251-2259

21 Poch Martell M, Sibai H, Deotare U, Lipton JH. Ponatinib in the therapy of chronic myeloid leukemia. Expert Rev Hematol 2016; 9(10):923-932

22 Douxfils J, Haguet H, Mullier F, Chatelain C, Graux C, Dogné JM. Association between BCR-ABL tyrosine kinase inhibitors for chronic myeloid leukemia and cardiovascular events, major molecular response, and overall survival: a systematic review and meta-analysis. JAMA Oncol 2016; doi: 10.1001/jamaoncol. 2015.5932 
23 Haguet H, Douxfils J, Mullier F, Chatelain C, Graux C, Dogné JM. Risk of arterial and venous occlusive events in chronic myeloid leukemia patients treated with new generation BCR-ABL tyrosine kinase inhibitors: a systematic review and meta-analysis. Expert Opin Drug Saf 2017;16(01):5-12

24 Cortes J, Kim D-W, Pinilla-Ibarz J, et al. Ponatinib efficacy and safety in heavily pretreated leukemia patients: 3-year results of the PACE trial. 20th Congress of EHA; Copenhagen, Denmark, June 12, 2015

25 Pasvolsky O, Leader A, Iakobishvili Z, Wasserstrum Y, Kornowski $\mathrm{R}$, Raanani P. Tyrosine kinase inhibitor associated vascular toxicity in chronic myeloid leukemia. Cardio-Oncology 2015;1(01):5

26 Gora-Tybor J, Medras E, Calbecka M, et al. Real-life comparison of severe vascular events and other non-hematological complications in patients with chronic myeloid leukemia undergoing second-line nilotinib or dasatinib treatment. Leuk Lymphoma 2015;56(08): 2309-2314

27 le Coutre PD, Hughes TP, Mahon FX, et al. Low incidence of peripheral arterial disease in patients receiving dasatinib in clinical trials. Leukemia 2016;30(07):1593-1596

28 Sam PY, Ahaneku H, Nogueras-Gonzalez GM, et al. Cardiovascular events among patients with chronic myeloid leukemia (CML) treated with tyrosine kinase inhibitors (TKIs). Blood 2016; 128(22):1919

29 Lassila M, Allen TJ, Cao Z, et al. Imatinib attenuates diabetesassociated atherosclerosis. Arterioscler Thromb Vasc Biol 2004; 24(05):935-942

$30 \mathrm{Ma}$ LK, Li Q He LF, et al. Imatinib attenuates myocardial fibrosis in association with inhibition of the PDGFR $\alpha$ activity. Arq Bras Cardiol 2012;99(06):1082-1091

31 Fossard G, Blond E, Balsat M, et al. Hyperhomocysteinemia and high doses of nilotinib favor cardiovascular events in chronic phase chronic myelogenous leukemia patients. Haematologica 2016;101(03):e86-e90

32 Kostos L, Burbury K, Srivastava G, Prince HM. Gastrointestinal bleeding in a chronic myeloid leukaemia patient precipitated by dasatinib-induced platelet dysfunction: case report. Platelets 2015;26(08):809-811

33 Mazharian A, Ghevaert C, Zhang L, Massberg S, Watson SP. Dasatinib enhances megakaryocyte differentiation but inhibits platelet formation. Blood 2011;117(19):5198-5206

34 Alhawiti N, Burbury KL, Kwa FA, et al. The tyrosine kinase inhibitor, nilotinib potentiates a prothrombotic state. Thromb Res 2016;145:54-64

35 Lotfi K, Deb S, Sjöström C, Tharmakulanathan A, Boknäs N, Ramström S. Individual variation in hemostatic alterations caused by tyrosine kinase inhibitors: a way to improve personalized cancer therapy? Blood 2016;128(22):1908

36 Li R, Grosser T, Diamond SL. Microfluidic whole blood testing of platelet response to pharmacological agents. Platelets 2017;28 (05):457-462

37 Senis YA, Mazharian A, Mori J. Src family kinases: at the forefront of platelet activation. Blood 2014;124(13):2013-2024

38 Gratacap MP, Martin V, Valéra MC, et al. The new tyrosine-kinase inhibitor and anticancer drug dasatinib reversibly affects platelet activation in vitro and in vivo. Blood 2009;114(09):1884-1892

39 Albrecht-Schgoer K, Huber K, Grebien F, et al. Nilotinib exerts direct pro-atherogenic and anti-angiogenic effects on vascular endothelial cells: a potential explanation for drug-induced vasculopathy in CML. Blood 2013;122(21):257

40 Shimabukuro-Vornhagen A, Rothe A, Nogova L, Kochanek M, Scheid C, von Bergwelt-Baildon M. Improvement of platelet dysfunction in chronic myelogenous leukemia following treatment with imatinib: a case report. J Med Case Reports 2011; 5:215

41 Neelakantan P, Marin D, Laffan M, Goldman J, Apperley J, Milojkovic D. Platelet dysfunction associated with ponatinib, a new pan BCR-ABL inhibitor with efficacy for chronic myeloid leukemia resistant to multiple tyrosine kinase inhibitor therapy. Haematologica 2012;97(09):1444

42 Loren CP, Aslan JE, Rigg RA, et al. The BCR-ABL inhibitor ponatinib inhibits platelet immunoreceptor tyrosine-based activation motif (ITAM) signaling, platelet activation and aggregate formation under shear. Thromb Res 2015;135(01):155-160

43 Rivera VM, Pritchard JR, Gonzalvez F, Baker T, Gozgit JM, Hodgson G. Comparative TKI profiling analyses to explore potential mechanisms of Ponatinib-associated arterial thrombotic events. Blood 2014;124(21):1783

44 Quintás-Cardama A, Han X, Kantarjian H, Cortes J. Tyrosine kinase inhibitor-induced platelet dysfunction in patients with chronic myeloid leukemia. Blood 2009;114(02):261-263

45 Breccia M, Muscaritoli M, Aversa Z, Mandelli F, Alimena G. Imatinib mesylate may improve fasting blood glucose in diabetic $\mathrm{Ph}+$ chronic myelogenous leukemia patients responsive to treatment. J Clin Oncol 2004;22(22):4653-4655

46 Breccia M, Alimena G. The metabolic consequences of imatinib mesylate: changes on glucose, lypidic and bone metabolism. Leuk Res 2009;33(07):871-875

47 Agostino NM, Chinchilli VM, Lynch CJ, et al. Effect of the tyrosine kinase inhibitors (sunitinib, sorafenib, dasatinib, and imatinib) on blood glucose levels in diabetic and nondiabetic patients in general clinical practice. J Oncol Pharm Pract 2011;17(03):197-202

48 Breccia M, Muscaritoli M, Cannella L, Stefanizzi C, Frustaci A, Alimena G. Fasting glucose improvement under dasatinib treatment in an accelerated phase chronic myeloid leukemia patient unresponsive to imatinib and nilotinib. Leuk Res 2008;32(10): $1626-1628$

49 Ono K, Suzushima H, Watanabe Y, et al. Rapid amelioration of hyperglycemia facilitated by dasatinib in a chronic myeloid leukemia patient with type 2 diabetes mellitus. Intern Med 2012;51(19):2763-2766

50 Louvet C, Szot GL, Lang J, et al. Tyrosine kinase inhibitors reverse type 1 diabetes in nonobese diabetic mice. Proc Natl Acad Sci U S A 2008;105(48):18895-18900

51 Hägerkvist R, Jansson L, Welsh N. Imatinib mesylate improves insulin sensitivity and glucose disposal rates in rats fed a high-fat diet. Clin Sci (Lond) 2008;114(01):65-71

52 Han MS, Chung KW, Cheon HG, et al. Imatinib mesylate reduces endoplasmic reticulum stress and induces remission of diabetes in $\mathrm{db} / \mathrm{db}$ mice. Diabetes 2009;58(02):329-336

53 Hägerkvist R, Makeeva N, Elliman S, Welsh N. Imatinib mesylate (Gleevec) protects against streptozotocin-induced diabetes and islet cell death in vitro. Cell Biol Int 2006;30(12):1013-1017

54 Hägerkvist R, Sandler S, Mokhtari D, Welsh N. Amelioration of diabetes by imatinib mesylate (Gleevec): role of beta-cell NFkappaB activation and anti-apoptotic preconditioning. FASEB J 2007;21(02):618-628

55 Xia CQ, Zhang P, Li S, et al. C-Abl inhibitor imatinib enhances insulin production by $\beta$ cells: $c$-Abl negatively regulates insulin production via interfering with the expression of $\mathrm{NKx} 2.2$ and GLUT-2. PLoS One 2014;9(05):e97694

56 Sadiq S, Owen E, Foster T, et al. Nilotinib-induced metabolic dysfunction: insights from a translational pilot study using in vitro adipocyte models and patient cohorts. 22nd EHA congress; June 22-25, 2017; Madrid, Spain

57 Iizuka K, Niwa H, Kato T, Takeda J. Dasatinib improves insulin sensitivity and affects lipid metabolism in a patient with chronic myeloid leukaemia. BMJ Case Rep 2016;2016:201

58 Fitter S, Vandyke K, Schultz CG, White D, Hughes TP, Zannettino AC. Plasma adiponectin levels are markedly elevated in imatinibtreated chronic myeloid leukemia (CML) patients: a mechanism for improved insulin sensitivity in type 2 diabetic CML patients? J Clin Endocrinol Metab 2010;95(08):3763-3767

59 Hosch SE, Olefsky JM, Kim JJ. APPLied mechanics: uncovering how adiponectin modulates insulin action. Cell Metab 2006;4 (01):5-6 
60 Lihn AS, Pedersen SB, Richelsen B. Adiponectin: action, regulation and association to insulin sensitivity. Obes Rev 2005;6(01): $13-21$

61 Fitter S, Vandyke K, Gronthos S, Zannettino AC. Suppression of PDGF-induced PI3 kinase activity by imatinib promotes adipogenesis and adiponectin secretion. J Mol Endocrinol 2012;48 (03):229-240

62 Aichberger KJ, Herndlhofer S, Schernthaner GH, et al. Progressive peripheral arterial occlusive disease and other vascular events during nilotinib therapy in CML. Am J Hematol 2011;86(07): 533-539

63 Breccia M, Muscaritoli M, Gentilini F, et al. Impaired fasting glucose level as metabolic side effect of nilotinib in non-diabetic chronic myeloid leukemia patients resistant to imatinib. Leuk Res 2007;31(12):1770-1772

64 Castagnetti F, Breccia M, Gugliotta G, et al; GIMEMA CML Working Party. Nilotinib $300 \mathrm{mg}$ twice daily: an academic single-arm study of newly diagnosed chronic phase chronic myeloid leukemia patients. Haematologica 2016;101(10):1200-1207

65 Delphine R, Gautier J-f, Breccia M, et al. Incidence of hyperglycemia by 3 years in patients (Pts) with newly diagnosed chronic myeloid leukemia in chronic phase (CML-CP) treated with Nilotinib (NIL) or Imatinib (IM) in ENESTnd. Blood 2012;120 (21):1686

66 Iurlo A, Orsi E, Cattaneo D, et al. Effects of first- and secondgeneration tyrosine kinase inhibitor therapy on glucose and lipid metabolism in chronic myeloid leukemia patients: a real clinical problem? Oncotarget 2015;6(32):33944-33951

67 Racil Z, Razga F, Drapalova J, et al. Mechanism of impaired glucose metabolism during nilotinib therapy in patients with chronic myelogenous leukemia. Haematologica 2013;98(10): e124-e126

68 Ito Y, Miyamoto T, Chong Y, Maki T, Akashi K, Kamimura T. Nilotinib exacerbates diabetes mellitus by decreasing secretion of endogenous insulin. Int J Hematol 2013;97(01):135-138

69 Hadzijusufovic E, Herndlhofer S, Aichberger KJ, et al. Nilotinib exerts direct effects on vascular endothelial cells and may act as a co-trigger of atherosclerosis in patients with $\mathrm{Ph}+\mathrm{CML}$. Blood 2011;118(21):2753

70 Breccia M, Loglisci G, Salaroli A, Serrao A, Alimena G. Nilotinibmediated increase in fasting glucose level is reversible, does not convert to type 2 diabetes and is likely correlated with increased body mass index. Leuk Res 2012;36(04):e66-e67

71 Saglio G, Larson RA, Hughes TP, et al. Efficacy and safety of Nilotinib in chronic phase (CP) chronic myeloid leukemia (CML) patients (Pts) with type 2 diabetes in the ENESTnd trial. Blood 2010;116(21):3430

72 Frasca F, Pandini G, Malaguarnera R, et al. Role of c-Abl in directing metabolic versus mitogenic effects in insulin receptor signaling. J Biol Chem 2007;282(36):26077-26088

73 Franceschino A, Tornaghi L, Benemacher V, Assouline S, Gambacorti-Passerini C. Alterations in creatine kinase, phosphate and lipid values in patients with chronic myeloid leukemia during treatment with imatinib. Haematologica 2008;93(02): 317-318

74 Gottardi M, Manzato E, Gherlinzoni F. Imatinib and hyperlipidemia. N Engl J Med 2005;353(25):2722-2723

75 Gologan R, Constantinescu G, Georgescu D, et al. Hypolipemiant besides antileukemic effect of imatinib mesylate. Leuk Res 2009; 33(09):1285-1287

76 Ellis M, Krashin E, Hamburger-Avnery O, Gan S, Elis A, AshurFabian O. The anti-leukemic and lipid lowering effects of imatinib are not hindered by statins in CML: a retrospective clinical study and in vitro assessment of lipid-genes transcription. Leuk Lymphoma 2017;58(05):1172-1177

77 Franklin M, Burns L, Perez S, Yerragolam D, Makenbaeva D. Incidence of type II diabetes mellitus and hyperlipidemia in patients prescribed Dasatinib or Nilotinib as first or second line therapy for chronic myelogenous leukemia (CML). Blood 2016; 128(22):4766

78 Rea D, Mirault T, Cluzeau T, et al. Early onset hypercholesterolemia induced by the 2nd-generation tyrosine kinase inhibitor nilotinib in patients with chronic phase-chronic myeloid leukemia. Haematologica 2014;99(07):1197-1203

79 Maurizot A, Beressi JP, Manéglier B, et al. Rapid clinical improvement of peripheral artery occlusive disease symptoms after nilotinib discontinuation despite persisting vascular occlusion. Blood Cancer J 2014;4:e247

80 Breccia M, Molica M, Alimena G. How tyrosine kinase inhibitors impair metabolism and endocrine system function: a systematic updated review. Leuk Res 2014;38(12):1392-1398

81 Venalis P, Maurer B, Akhmetshina A, et al. Lack of inhibitory effects of the anti-fibrotic drug imatinib on endothelial cell functions in vitro and in vivo. J Cell Mol Med 2009;13(10): 4185-4191

82 Gover-Proaktor A, Granot G, Shapira S, et al. Ponatinib reduces viability, migration, and functionality of human endothelial cells. Leuk Lymphoma 2017;58(06):1455-1467

83 Vallières K, Petitclerc E, Laroche G. On the ability of imatinib mesylate to inhibit smooth muscle cell proliferation without delaying endothelialization: an in vitro study. Vascul Pharmacol 2009;51(01):50-56

84 Hacker TA, Griffin MO, Guttormsen B, Stoker S, Wolff MR. Platelet-derived growth factor receptor antagonist STI571 (imatinib mesylate) inhibits human vascular smooth muscle proliferation and migration in vitro but not in vivo. J Invasive Cardiol 2007;19(06):269-274

85 Vrekoussis T, Stathopoulos EN, De Giorgi U, et al. Modulation of vascular endothelium by imatinib: a study on the EA.hy 926 endothelial cell line. J Chemother 2006;18(01):56-65

86 Letsiou E, Rizzo AN, Sammani S, et al. Differential and opposing effects of imatinib on LPS- and ventilator-induced lung injury. Am J Physiol Lung Cell Mol Physiol 2015;308(03):L259-L269

87 Guignabert C, Phan C, Seferian A, et al. Dasatinib induces lung vascular toxicity and predisposes to pulmonary hypertension. J Clin Invest 2016;126(09):3207-3218

88 Rizzo AN, Sammani S, Esquinca AE, et al. Imatinib attenuates inflammation and vascular leak in a clinically relevant two-hit model of acute lung injury. Am J Physiol Lung Cell Mol Physiol 2015;309(11):L1294-L1304

89 Katgı A, Sevindik ÖG, Gökbulut AA, et al. Nilotinib does not alter the secretory functions of carotid artery endothelial cells in a prothrombotic or antithrombotic fashion. Clin Appl Thromb Hemost 2015;21(07):678-683

90 Hadzijusufovic E, Kirchmair R, Theurl M, et al. Ponatinib exerts multiple effects on vascular endothelial cells: possible mechanisms and explanations for the adverse vascular events seen in CML patients treated with Ponatinib. Blood 2016;128 (22):1883

91 Lorenzi M, Cagliero E, Toledo S. Glucose toxicity for human endothelial cells in culture. Delayed replication, disturbed cell cycle, and accelerated death. Diabetes 1985;34(07):621-627

92 Shah NP, Wallis N, Farber HW, et al. Clinical features of pulmonary arterial hypertension in patients receiving dasatinib. Am J Hematol 2015;90(11):1060-1064

93 Frost AE, Barst RJ, Hoeper MM, et al. Long-term safety and efficacy of imatinib in pulmonary arterial hypertension. J Heart Lung Transplant 2015;34(11):1366-1375

94 Dasgupta SK, Le A, Vijayan KV, Thiagarajan P. Dasatinib inhibits actin fiber reorganization and promotes endothelial cell permeability through RhoA-ROCK pathway. Cancer Med 2017;6(04): 809-818

95 Dewar AL, Domaschenz RM, Doherty KV, Hughes TP, Lyons AB. Imatinib inhibits the in vitro development of the monocyte/ macrophage lineage from normal human bone marrow progenitors. Leukemia 2003;17(09):1713-1721 
96 Brownlow N, Mol C, Hayford C, Ghaem-Maghami S, Dibb NJ. Dasatinib is a potent inhibitor of tumour-associated macrophages, osteoclasts and the FMS receptor. Leukemia 2009;23 (03):590-594

97 Dewar AL, Doherty KV, Hughes TP, Lyons AB. Imatinib inhibits the functional capacity of cultured human monocytes. Immunol Cell Biol 2005;83(01):48-56

98 Dewar AL, Cambareri AC, Zannettino ACW, et al. Macrophage colony-stimulating factor receptor c-fms is a novel target of imatinib. Blood 2005;105(08):3127-3132

99 Metharom P, Martin K, Kumar AH, et al. Pleiotropic role for monocyte $\mathrm{C}$-fms protein in response to vascular injury: potential therapeutic target. Atherosclerosis 2011;216(01):74-82

100 Gacic J, Vorkapic E, Olsen RS, et al. Imatinib reduces cholesterol uptake and matrix metalloproteinase activity in human THP-1 macrophages. Pharmacol Rep 2016;68(01):1-6

101 Cwynarski K, Laylor R, Macchiarulo E, et al. Imatinib inhibits the activation and proliferation of normal T lymphocytes in vitro. Leukemia 2004;18(08):1332-1339

102 Brownlow N, Russell AE, Saravanapavan H, et al. Comparison of nilotinib and imatinib inhibition of FMS receptor signaling, macrophage production and osteoclastogenesis. Leukemia 2008;22(03):649-652

103 Ozanne J, Prescott AR, Clark K. The clinically approved drugs dasatinib and bosutinib induce anti-inflammatory macrophages by inhibiting the salt-inducible kinases. Biochem J 2015;465 (02):271-279

104 Darling NJ, Toth R, Arthur JS, Clark K. Inhibition of SIK2 and SIK3 during differentiation enhances the anti-inflammatory phenotype of macrophages. Biochem J 2017;474(04):521-537

105 Schade AE, Schieven GL, Townsend R, et al. Dasatinib, a smallmolecule protein tyrosine kinase inhibitor, inhibits T-cell activation and proliferation. Blood 2008;111(03):1366-1377

106 Blake SJ, Lyons AB, Hughes TP. Nilotinib inhibits the Src-family kinase LCK and T-cell function in vitro. J Cell Mol Med 2009;13 (03):599-601

107 Blake S, Hughes TP, Mayrhofer G, Lyons AB. The Src/ABL kinase inhibitor dasatinib (BMS-354825) inhibits function of normal human T-lymphocytes in vitro. Clin Immunol 2008;127(03): 330-339

108 Lee KC, Ouwehand I, Giannini AL, Thomas NS, Dibb NJ, Bijlmakers MJ. Lck is a key target of imatinib and dasatinib in T-cell activation. Leukemia 2010;24(04):896-900

109 Farha S, Dweik R, Rahaghi F, et al. Imatinib in pulmonary arterial hypertension: c-Kit inhibition. Pulm Circ 2014;4(03):452-455

110 Juurikivi A, Sandler C, Lindstedt KA, et al. Inhibition of c-kit tyrosine kinase by imatinib mesylate induces apoptosis in mast cells in rheumatoid synovia: a potential approach to the treatment of arthritis. Ann Rheum Dis 2005;64(08): $1126-1131$

111 Sun YL, Kumar P, Sodani K, et al. Ponatinib enhances anticancer drug sensitivity in MRP7-overexpressing cells. Oncol Rep 2014; 31(04):1605-1612

112 Yvan-Charvet L, Wang N, Tall AR. Role of HDL, ABCA1, and ABCG1 transporters in cholesterol efflux and immune responses. Arterioscler Thromb Vasc Biol 2010;30(02):139-143

113 Chen Z, Lee FY, Bhalla KN, Wu J. Potent inhibition of plateletderived growth factor-induced responses in vascular smooth muscle cells by BMS-354825 (dasatinib). Mol Pharmacol 2006; 69(05):1527-1533

114 Nakamura K, Akagi S, Ogawa A, et al. Pro-apoptotic effects of imatinib on PDGF-stimulated pulmonary artery smooth muscle cells from patients with idiopathic pulmonary arterial hypertension. Int J Cardiol 2012;159(02):100-106

115 Li L, Blumenthal DK, Masaki T, Terry CM, Cheung AK. Differential effects of imatinib on PDGF-induced proliferation and PDGF receptor signaling in human arterial and venous smooth muscle cells. J Cell Biochem 2006;99(06):1553-1563
116 Rocha A, Azevedo I, Soares R. Anti-angiogenic effects of imatinib target smooth muscle cells but not endothelial cells. Angiogenesis 2007;10(04):279-286

117 Ballinger ML, Osman N, Hashimura K, et al. Imatinib inhibits vascular smooth muscle proteoglycan synthesis and reduces LDL binding in vitro and aortic lipid deposition in vivo. J Cell Mol Med 2010;14(6B):1408-1418

118 Distler JH, Jüngel A, Huber LC, et al. Imatinib mesylate reduces production of extracellular matrix and prevents development of experimental dermal fibrosis. Arthritis Rheum 2007;56(01): 311-322

119 Yoshiji H, Noguchi R, Kuriyama S, et al. Imatinib mesylate (STI571) attenuates liver fibrosis development in rats. Am J Physiol Gastrointest Liver Physiol 2005;288(05):G907-G913

120 Salhotra A, Tsai N, Thomas SH, et al. Sclerodermatous chronic GVHD in patients receiving tyrosine kinase inhibitors after allogeneic hematopoietic cell transplantation. Bone Marrow Transplant 2015;50(01):139-141

121 Makiyama Y, Toba K, Kato K, et al. Imatinib mesilate inhibits neointimal hyperplasia via growth inhibition of vascular smooth muscle cells in a rat model of balloon injury. Tohoku J Exp Med 2008;215(04):299-306

122 Park D, Kim SM, Min SI, Ha J, Kim IG, Min SK. Inhibition of intimal hyperplasia by local perivascular application of rapamycin and imatinib mesilate after carotid balloon injury. J Korean Surg Soc 2013;85(06):296-301

123 Leppänen $\mathrm{O}$, Rutanen J, Hiltunen $\mathrm{MO}$, et al. Oral imatinib mesylate (STI571/Gleevec) improves the efficacy of local intravascular vascular endothelial growth factor-C gene transfer in reducing neointimal growth in hypercholesterolemic rabbits. Circulation 2004;109(09):1140-1146

124 Sevim KZ, Silistreli O, Gorgu M, Sevim O, Ergur B. Short-term vasculoprotective effects of imatinib mesylate on intimal hyperplasia of arterial anastomosis: An experimental study using a rabbit model. Can J Plast Surg 2012;20(04):223-228

$125 \mathrm{Wu}$ J, Chen Z, Bhalla KN. Treatment of restenosis and stenosis with dasatinib (2014). USF Patents. 152

126 Sánchez-Ortega I, Servitje O, Arnan M, et al. Dasatinib as salvage therapy for steroid refractory and imatinib resistant or intolerant sclerotic chronic graft-versus-host disease. Biol Blood Marrow Transplant 2012;18(02):318-323

127 Marinelli Busilacchi E, Olivieri J, Viola N, et al. The exaggerated collagen expression in Gvhd-fibroblasts is effectively inhibited by therapeutic concentration of Nilotinib. Blood 2016;128(22): 4597

128 Shiha GE, Abu-Elsaad NM, Zalata KR, Ibrahim TM. Tracking antifibrotic pathways of nilotinib and imatinib in experimentally induced liver fibrosis: an insight. Clin Exp Pharmacol Physiol 2014;41(10):788-797

129 Giles FJ, O’Dwyer M, Swords R. Class effects of tyrosine kinase inhibitors in the treatment of chronic myeloid leukemia. Leukemia 2009;23(10):1698-1707

130 Rix U, Hantschel O, Dürnberger G, et al. Chemical proteomic profiles of the BCR-ABL inhibitors imatinib, nilotinib, and dasatinib reveal novel kinase and nonkinase targets. Blood 2007;110 (12):4055-4063

131 Pardanani A, Tefferi A. Imatinib targets other than bcr/abl and their clinical relevance in myeloid disorders. Blood 2004;104 (07):1931-1939

132 Bantscheff M, Eberhard D, Abraham Y, et al. Quantitative chemical proteomics reveals mechanisms of action of clinical ABL kinase inhibitors. Nat Biotechnol 2007;25(09):10351044

133 Weisberg E, Manley PW, Breitenstein W, et al. Characterization of AMN107, a selective inhibitor of native and mutant Bcr-Abl. Cancer Cell 2005;7(02):129-141

134 Buchdunger E, Cioffi CL, Law N, et al. Abl protein-tyrosine kinase inhibitor STI571 inhibits in vitro signal transduction mediated 
by c-kit and platelet-derived growth factor receptors. J Pharmacol Exp Ther 2000;295(01):139-145

135 Manley PW, Drueckes P, Fendrich G, et al. Extended kinase profile and properties of the protein kinase inhibitor nilotinib. Biochim Biophys Acta 2010;1804(03):445-453

136 Fabian MA, Biggs WH III, Treiber DK, et al. A small moleculekinase interaction map for clinical kinase inhibitors. Nat Biotechnol 2005;23(03):329-336

137 Shi H, Zhang CJ, Chen GY, Yao SQ. Cell-based proteome profiling of potential dasatinib targets by use of affinity-based probes. J Am Chem Soc 2012;134(06):3001-3014

138 Liu Y, Wang Z, Kwong SQ et al. Inhibition of PDGF, TGF- $\beta$, and Abl signaling and reduction of liver fibrosis by the small molecule Bcr-Abl tyrosine kinase antagonist Nilotinib. J Hepatol 2011;55 (03):612-625

139 Sakamoto A, Sugamoto Y, Tokunaga Y, et al. Expression profiling of the ephrin (EFN) and Eph receptor (EPH) family of genes in atherosclerosis-related human cells. J Int Med Res 2011;39(02): 522-527

140 Khan AA, Sandhya VK, Singh P, et al. Signaling network map of endothelial TEK tyrosine kinase. J Signal Transduct 2014; 2014:173026

141 Chislock EM, Ring C, Pendergast AM. Abl kinases are required for vascular function, Tie2 expression, and angiopoietin-1-mediated survival. Proc Natl Acad Sci U S A 2013;110(30):12432-12437

142 Boulanger CM, Loyer X, Rautou PE, Amabile N. Extracellular vesicles in coronary artery disease. Nat Rev Cardiol 2017;14 (05):259-272

143 Aghel N, Delgado DH, Lipton JH. Cardiovascular toxicities of BCRABL tyrosine kinase inhibitors in chronic myeloid leukemia: preventive strategies and cardiovascular surveillance. Vasc Health Risk Manag 2017;13:293-303

144 Damrongwatanasuk R, Fradley MG. Cardiovascular complications of targeted therapies for chronic myeloid leukemia. Curr Treat Options Cardiovasc Med 2017;19(04):24

145 Valent P, Hadzijusufovic E, Hoermann G, et al. Risk factors and mechanisms contributing to TKI-induced vascular events in patients with CML. Leuk Res 2017;59:47-54

146 García-Gutiérrez V, Jiménez-Velasco A, Gómez-Casares MT, Sánchez-Guijo F, López-Sendón JL, Steegmann Olmedillas JL. [Cardiovascular management of patients with chronic myeloid leukemia from a multidisciplinary perspective, and proposing action protocol by consensus meeting]. Med Clin (Barc) 2016; 146(12):561.e1-561.e8
147 Aman J, van Bezu J, Damanafshan A, et al. Effective treatment of edema and endothelial barrier dysfunction with imatinib. Circulation 2012;126(23):2728-2738

148 Kim SR, Suh W. Beneficial effects of the Src inhibitor, dasatinib, on breakdown of the blood-retinal barrier. Arch Pharm Res 2017; 40(02):197-203

149 Sukegawa M, Wang X, Nishioka C, et al. The BCR/ABL tyrosine kinase inhibitor, nilotinib, stimulates expression of IL-1 $\beta$ in vascular endothelium in association with downregulation of miR-3p. Leuk Res 2017;58:83-90

150 Wolf AM, Wolf D, Rumpold H, et al. The kinase inhibitor imatinib mesylate inhibits TNF-alpha production in vitro and prevents TNF-dependent acute hepatic inflammation. Proc Natl Acad Sci U S A 2005;102(38):13622-13627

151 Fraser CK, Lousberg EL, Kumar R, Hughes TP, Diener KR, Hayball JD. Dasatinib inhibits the secretion of TNF-alpha following TLR stimulation in vitro and in vivo. Exp Hematol 2009;37(12): $1435-1444$

152 Seggewiss R, Loré K, Greiner E, et al. Imatinib inhibits T-cell receptor-mediated T-cell proliferation and activation in a dosedependent manner. Blood 2005;105(06):2473-2479

153 Dietz AB, Souan L, Knutson GJ, Bulur PA, Litzow MR, Vuk-Pavlovic $\mathrm{S}$. Imatinib mesylate inhibits T-cell proliferation in vitro and delayed-type hypersensitivity in vivo. Blood 2004;104(04): 1094-1099

154 Chen J, Schmitt A, Chen B, et al. Nilotinib hampers the proliferation and function of CD8 $+\mathrm{T}$ lymphocytes through inhibition of $\mathrm{T}$ cell receptor signalling. J Cell Mol Med 2008;12 (5B):2107-2118

155 Perros F, Montani D, Dorfmüller P, et al. Platelet-derived growth factor expression and function in idiopathic pulmonary arterial hypertension. Am J Respir Crit Care Med 2008;178(01):81-88

156 Canning P, Tan L, Chu K, Lee SW, Gray NS, Bullock AN. Structural mechanisms determining inhibition of the collagen receptor DDR1 by selective and multi-targeted type II kinase inhibitors. J Mol Biol 2014;426(13):2457-2470

157 Hantschel O, Rix U, Superti-Furga G. Target spectrum of the BCRABL inhibitors imatinib, nilotinib and dasatinib. Leuk Lymphoma 2008;49(04):615-619

158 Remsing Rix LL, Rix U, Colinge J, et al. Global target profile of the kinase inhibitor bosutinib in primary chronic myeloid leukemia cells. Leukemia 2009;23(03):477-485

159 Green MR, Newton MD, Fancher KM. Off-Target Effects of BCRABL and JAK2 Inhibitors. Am J Clin Oncol 2016;39(01):76-84 